\title{
PARABOLIC AND HYPERBOLIC SCREW MOTION SURFACES IN $\mathbb{H}^{2} \times \mathbb{R}$

\author{
RICARDO SA EARP
}

(Received 22 May 2006; accepted 8 August 2006)

Communicated by C. D. Hodgson

\begin{abstract}
In this paper we find many families in the product space $\mathbb{H}^{2} \times \mathbb{R}$ of complete embedded, simply connected, minimal and surfaces with constant mean curvature $H$ such that $|H| \leq 1 / 2$. We study complete surfaces invariant either by parabolic or by hyperbolic screw motions. We study the notion of isometric associate immersions. We exhibit an explicit formula for a Scherk-type minimal surface. We give a one-parameter family of entire vertical graphs of mean curvature $1 / 2$. We prove a generalized Bour lemma that can be applied to $\mathbb{H}^{2} \times \mathbb{R}, \mathbb{S}^{2} \times \mathbb{R}$ and to Heisenberg's space to produce a family of screw motion surfaces isometric to a given one.
\end{abstract}

2000 Mathematics subject classification: 53C42.

Keywords and phrases: minimal and constant mean curvature surfaces, isometric, associate, embedded, vertical and horizontal graphs.

\section{Introduction}

In a pioneering paper, Rosenberg [17] studied minimal surfaces in $M^{2} \times \mathbb{R}$, where $M^{2}$ is a round sphere, a complete Riemannian surface with a metric of nonnegative curvature, or $M^{2}=\mathbb{H}^{2}$, the hyperbolic plane. He opened a quite interesting new branch of research in surface theory, stimulating several works on the subject. The main scope of the present paper is to discover complete embedded minimal and constant mean curvature surfaces. Now, we briefly summarize our results, as follows.

We will study minimal and constant mean curvature surfaces in $\mathbb{H}^{2} \times \mathbb{R}$, invariant by parabolic screw motions, that is, invariant by a one-parameter group of isometries such that each element is given by the composition of a parabolic translation with a vertical translation. We will find a two-parameter family of complete embedded, simply connected, minimal surfaces which contains surfaces invariant by parabolic translations. We also will obtain a one-parameter family of complete, embedded,

The author would like to thank CNPq and PRONEX of Brazil, for partial financial support.

(c) 2008 Australian Mathematical Society 1446-7887/08 \$A2.00+0.00 


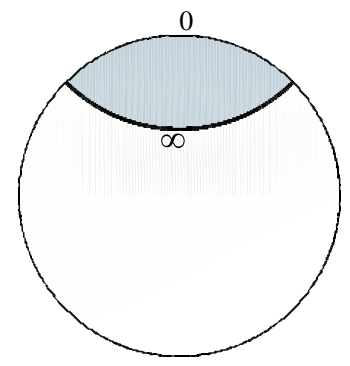

FIGURE 1. A ball model for $\mathbb{H}^{2} \times\{0\}$.

simply connected, stable minimal surfaces which contains the hyperbolic plane $\mathbb{H}^{2} \times\{0\}$. We will then construct a two-parameter family of complete $H$-surfaces, that is, surfaces with constant mean curvature $H$, such that $|H|<1 / 2$, simply connected and embedded, which contains a surface invariant by parabolic translations. We will find in this family explicit nonparametric formulas for a one-parameter subfamily of complete, embedded, stable, $H$-surfaces $(|H|<1 / 2)$. Furthermore, we will exhibit a one-parameter family of minimal and constant mean curvature surfaces $(H=1 / 2)$, invariant by hyperbolic screw motions. We also obtain minimal and $H$-surfaces $(|H|<1 / 2)$ invariant by hyperbolic translations. Each such surface is complete, embedded, simply connected, and stable. We note that there are no stable $H$-surfaces, complete and noncompact, for $H>1 / \sqrt{3}$. This is a result of Nelli and Rosenberg [15].

Furthermore, we will derive an explicit simple nonparametric formula for a Scherktype minimal surface, invariant by hyperbolic translations, as found independently by Abresch (private communication). Abresch and Rosenberg have applied it as a barrier studying the Dirichlet problem for the minimal surface equation in $\mathbb{H}^{2} \times \mathbb{R}$. We will explain their geometric construction later on in the text. We observe that this Scherktype surface can be seen as a complete vertical graph over a domain in $\mathbb{H}^{2}$ taking $\pm \infty$ value boundary data on a geodesic and zero (or constant) asymptotic value boundary data, that is, taking zero on an arc of the circle at infinity. See the shaded domain in Figure 1. In a paper with Toubiana [19] the current author studied surfaces in $\mathbb{H}^{2} \times \mathbb{R}$ and $\mathbb{S}^{2} \times \mathbb{R}$ invariant by standard screw motions, that is, a one-parameter group of isometries such that each element is the composition of a rotation around the vertical axis with a vertical translation. In that paper the authors obtained for $\ell>1 / \sqrt{2}$ a complete embedded simply connected minimal screw motion surface in $\mathbb{H}^{2} \times \mathbb{R}$ with pitch $\ell$. If $\ell=1$, each such surface has Gaussian curvature $K \equiv-1$ (see also [11, Example 18, p. 284]).

The current author working with Toubiana and Hauswirth established a uniqueness theorem in $\mathbb{H}^{2} \times \mathbb{R}$, or $\mathbb{S}^{2} \times \mathbb{R}$ [11]. They proved that the conformal metric and the related holomorphic Hopf function, arising from the theory of minimal immersions and conformal mappings, determine a minimal conformal immersion, up to an isometry of ambient space. They proved an existence theorem that produces the existence of the minimal associate family as a corollary. They also generalized a theorem by Krust (see [6, p. 118]) which states that an associate surface of a minimal 
vertical graph on a convex domain is a vertical graph. This theorem is true in $\mathbb{M} \times \mathbb{R}$ when the Gaussian curvature satisfies $K_{\mathbb{M}} \leq 0$; see [11].

Now let us make some more detailed comments about the present paper. Our idea is simple: we will consider $\mathbb{H}^{2}=\{x+i y, y>0\}$, the upper half-plane model of the hyperbolic plane, and we will consider the product space $\mathbb{H}^{2} \times \mathbb{R}$, with coordinates $(x, y, t)$, endowed with the metric $d \sigma^{2}=\left(d x^{2} / y^{2}\right)+\left(d y^{2} / y^{2}\right)+d t^{2}$. We will search for surfaces invariant by parabolic screw motions - a parabolic translation is identified with a horizontal Euclidean translation in this model. That is, we will study immersions of the form

$$
X(x, y)=(x, y, \lambda(y)+\ell x)
$$

Thus we will search for minimal and for constant mean curvature surfaces generated by applying Euclidean translations to the vertical graph $t=\lambda(y)$ lying in the $(y, t)$ vertical plane along the directions of the vector $(1,0, \ell)$. We say that $\ell$ is the pitch. When $\ell=0$, we obtain a surface invariant by parabolic translations. Of course, in this model this is related with two notions of nonparametric graphs. We will introduce them now for motivation. First, let us consider prescribed mean curvature horizontal $\mathrm{H}$ graphs given by $y=g(x, t)$, where $g(x, t)$ is a positive $C^{2}$ function. A computation shows that the horizontal mean curvature equation in $\mathbb{H}^{2} \times \mathbb{R}$ is given by

$$
\begin{aligned}
\frac{2 H}{g^{2}}\left(g_{t}^{2}+g^{2}\left(1+g_{x}^{2}\right)\right)^{3 / 2}= & g_{x x}\left(g^{2}+g_{t}^{2}\right)+g_{t t}\left(1+g_{x}^{2}\right) \\
& -2 g_{x} g_{t} g_{x t}+g\left(1+g_{x}^{2}\right) .
\end{aligned}
$$

An interesting question that arises is the Bernstein problem for these graphs: Are entire $H$-graphs invariant under parabolic screw motions?

Some classical constant mean curvature surfaces in $\mathbb{H}^{2} \times \mathbb{R}$ that arise naturally from this nonparametric point of view are related to this question: indeed, note that when $g$ is constant we obtain the vertical cylinder over a horocycle of mean curvature $1 / 2$. If $g_{t} \equiv 0$, we obtain the minimal vertical cylinder over a geodesic, the constant mean curvature vertical cylinder over an equidistant curve $(|H|<1 / 2)$, and the constant mean curvature vertical cylinder over a circle $(|H|<1 / 2)$. If $H$ is constant and $g_{x} \equiv 0$, we obtain the Abresch-Rosenberg [1] surface with mean curvature $H$, where $|H|<1 / 2$ given by $y=e^{t / a}, a=-2 H / \sqrt{1-4 H^{2}}$. Note that if $H=0$ and $g_{x} \equiv 0$, we can obtain a very simple formula for a one-parameter family of minimal stable complete surfaces invariant by a parabolic motion $y=$ $(\sin t) / d, d \neq 0$. This formula has already been established by Daniel [2] and this surface was known to Hauswirth [10]. We will construct a two-parameter family $y=g_{H}(x, t, d, \ell), d \neq 0$ of horizontal minimal graphs, which produces complete, simply connected and embedded minimal surfaces. If $\ell=0$, we rediscover the DanielHauswirth minimal surface. Fixing the parameter $d$ and varying $\ell$ we get a family of nonisometric deformations of Daniel-Hauswirth minimal surfaces, that is, two surfaces with different values of pitch $\ell$ are nonisometric. 
Let us now turn attention to vertical $H$-graphs given by a $C^{2}$ function $t=u(x, y)$. The vertical mean curvature equation in $\mathbb{H}^{2} \times \mathbb{R}$ is given by the following equation:

$$
\operatorname{div}_{\mathbb{H}}\left(\frac{\nabla_{\mathbb{H}} u}{W_{u}}\right)=2 H,
$$

where $\operatorname{div}_{\mathbb{H}}$ and $\nabla_{\mathbb{H}}$ are the hyperbolic divergence and gradient, respectively, and $W_{u}=\sqrt{1+\left|\nabla_{\mathbb{H}} u\right|_{\mathbb{H}}^{2}},|\cdot| \mathbb{H}$ being the norm in $\mathbb{H}^{2}$.

Gradient interior estimates and infinite boundary value problems for $H$-vertical graphs were inferred by Spruck [21] and Hauswirth et al. [12].

Consider the half-space model for $\mathbb{H}^{2}$, with Euclidean coordinates $x, y, y>0$. In such a model, the above equation takes the following form:

$$
\begin{aligned}
\frac{2 H}{y^{2}}\left(1+y^{2} u_{x}^{2}+y^{2} u_{y}^{2}\right)^{3 / 2}= & \left(1+y^{2} u_{x}^{2}\right) u_{y y}+\left(1+y^{2} u_{y}^{2}\right) u_{x x} \\
& -2 y^{2} u_{x} u_{y} u_{x y}-y u_{y}\left(u_{x}^{2}+u_{y}^{2}\right) .
\end{aligned}
$$

Of course, on account of (3), the above equation is a second-order quasilinear elliptic equation of divergence form, namely

$$
\operatorname{div}\left(\frac{\nabla u}{\sqrt{1+y^{2}|\nabla u|^{2}}}\right)=\frac{2 H}{y^{2}},
$$

where $\nabla u$ stands for the Euclidean gradient. If $t=a \ln y$, we rediscover the AbreschRosenberg surface given above. Notice that the tilted Euclidean plane $t=\ell x, y>0$, $\ell \neq 0$ gives rise to a solution of (4). It turns out that, for $\ell=1$, we will obtain a conjugate of a Scherk-type minimal surface. If $\ell=0$, we obtain the totally geodesic hyperbolic plane $\mathbb{H}^{2} \times\{0\}$. Note that we obtain a one-parameter family of minimal, nontotally geodesic stable complete surfaces in $\mathbb{H}^{2} \times \mathbb{R}$ foliated by geodesics of the ambient space (different from the helicoids) invariant by parabolic screw motions. Varying $\ell$, we have a family of nonisometric deformations of the hyperbolic plane, that is, two elements of this family with different pitches $\ell$ are nonisometric.

We will give an explicit formula for a one-parameter family of vertical $H$-graphs, $t=u_{H}(x, y, \ell)$, in $\mathbb{H}^{2} \times \mathbb{R}$ over the entire $\mathbb{H}^{2}$ with pitch $\ell$ and constant mean curvature $H$, where $|H|<1 / 2$. Varying $\ell$, we obtain a family of nonisometric simply connected, embedded and stable deformations of Abresch-Rosenberg surfaces $(\ell=0)$.

In fact, we will construct a two-parameter family of horizontal graphs $y=$ $g_{H}(x, t, d, \ell)$, with constant mean curvature $H$, where $|H|<1 / 2$, over the whole $x t$ plane that contains the above family; by setting $d=0$ we get the family $t=$ $u_{\mathcal{H}}(x, y, \ell)$ mentioned above. Again, fixing $d$ and varying $\ell$, we obtain a family of nonisometric deformations.

Moreover, we will obtain vertical minimal and $H$-graphs $(|H| \leq 1 / 2)$ over the entire hyperbolic plane invariant by hyperbolic screw motions (with pitch $\ell$ ). 
Incidentally, we will give an explicit, simple, nonparametric formula for a family of minimal entire vertical graphs invariant by hyperbolic screw motions.

We also exhibit a one-parameter family of entire vertical graphs of mean curvature $1 / 2$. Rosenberg posed the question of the uniqueness of these vertical graphs in the class of all 1/2-entire graphs. This is the 'Bernstein Problem' for $1 / 2$-surfaces in $\mathbb{H}^{2} \times \mathbb{R}$. We note that very recently Fernández and Mira classified the entire minimal graphs in Heisenberg space $\mathrm{Nil}_{3}$ [9]. This is related to the Bernstein problem cited before.

We would like to remark that the notions of horizontal and vertical graphs have appeared in the study of $H$-surfaces in hyperbolic space (see, for instance, [18]).

Finally, let $M^{2}$ be a two-dimensional Riemannian manifold. Assume the existence of a one-parameter group $\Gamma$ of isometries acting on $M^{2}$. We will say that $S$ is a $\Gamma$-screw motion surface if it is invariant by successive compositions of an element of $\Gamma$ with a vertical translation. If $\Gamma$ consists of rotations about the vertical axis we will say that this is a standard screw motion, instead of a $\Gamma$-screw motion. If $M^{3}=\mathbb{H}^{2} \times \mathbb{R}$, and $\Gamma$ is the group of parabolic translations we say that they are parabolic screw motions; if $\Gamma$ is the group of hyperbolic translations we say that they are hyperbolic screw motions. We will prove a generalized Bour lemma which is general enough to be applied to $\mathbb{H}^{2} \times \mathbb{R}$ and $\mathbb{S}^{2} \times \mathbb{R}$ (and to Heisenberg space). Given a $\Gamma$-screw motion surface $S$ we will obtain a two-parameter family of isometric $\Gamma$-screw motion surfaces to $S$, say $\mathcal{F}(\ell, m), m \neq 0$. In the case of parabolic screw motions in $\mathbb{H}^{2} \times \mathbb{R}$ this family contains a parabolic translation. The same is true for hyperbolic screw motions in $\mathbb{H}^{2} \times \mathbb{R}$. The case of standard screw motions is treated in [19]. More precisely, we find natural parameters $(s, \tau)$, so that the metric is of the form $d \sigma^{2}=d s^{2}+U^{2}(s) d \tau^{2}$, and we are able to describe entirely any screw motion surface in terms of the parameters $\ell, m$, and the metric determined by $U^{2}$. This can also be applied to Heisenberg space. In this paper, we will apply the Bour generalized lemma to screw motion surfaces in $\mathbb{H}^{2} \times \mathbb{R}$, to show that any two minimal isometric parabolic screw motion immersions are associate; that is, the absolute values of their Hopf functions are equal. The current author with Toubiana proved that this result also holds for standard screw motion surfaces in $\mathbb{H}^{2} \times \mathbb{R}$ and $\mathbb{S}^{2} \times \mathbb{R}$. Moreover, these authors in the same paper proved that, in $\mathbb{H}^{2} \times \mathbb{R}$, a catenoid is conjugate to a helicoid of pitch $\ell<1$. In this paper, we deduce that if $\ell=1$ then the helicoid is conjugate to a Daniel-Hauswirth minimal surface. Daniel has proved this result by another approach in [2]. It follows from his work that the helicoid of pitch $\ell>1$ is conjugate to a surface invariant by hyperbolic isometries. We will deduce this fact by outlining an alternative proof. Furthermore, we show that each such helicoid is associate to a parabolic screw motion surface. On the other hand, there exist families of isometric associate hyperbolic screw motion immersions, but there also exist isometric nonassociate hyperbolic screw motion immersions. Each parabolic screw motion surface is associate to a hyperbolic screw motion surface. For other relevant papers on this subject, the reader is referred to Meeks and Rosenberg [13], Nelli and Rosenberg [14], and Fernández and Mira [8]. 


\section{Complete embedded minimal and constant mean curvature surfaces in $\mathbb{H}^{2} \times \mathbb{R}$ invariant by parabolic screw motions}

Recall that we consider the product $\mathbb{H}^{2} \times \mathbb{R}$ (here $\mathbb{H}^{2}$ is the upper half-plane model) with coordinates $(x, y, t)$, endowed with the metric $d \sigma^{2}=d x^{2} / y^{2}+d y^{2} / y^{2}+d t^{2}$, where the inner product is denoted by $\langle\cdot, \cdot\rangle$ and the norm is denoted by $\|\cdot, \cdot\|$. We now turn our attention to nonparametric vertical graphs $X:(x, y) \mapsto(x, y, u(x, y))$, given by $C^{2}$ functions $t=u(x, y)$ over $\mathbb{H}^{2}$. Let $\widetilde{\nabla}$ be the Riemannian connection on $\mathbb{H}^{2} \times \mathbb{R}$. Let $X_{x}, X_{y}$ be the coordinate global frame field to the graph. It is straightforward to deduce that the upper unit normal $N$ is given by

$$
N=\frac{1}{\sqrt{1+y^{2}\left(u_{x}^{2}+u_{y}^{2}\right)}}\left(-u_{x} y^{2},-u_{y} y^{2}, 1\right) .
$$

We can also easily deduce that the connection is determined by the formulas

$$
\widetilde{\nabla}_{X_{x}} X_{x}=\left(0,1 / y, u_{x x}\right), \quad \widetilde{\nabla}_{X_{y}} X_{y}=\left(0,-1 / y, u_{y y}\right), \quad \widetilde{\nabla}_{X_{y}} X_{x}=\left(-1 / y, 0, u_{x y}\right) .
$$

We have therefore that the coefficients of the second fundamental form $l:=$ $\left\langle\widetilde{\nabla}_{X_{x}} X_{x}, N\right\rangle, n:=\left\langle\widetilde{\nabla}_{X_{y}} X_{y}, N\right\rangle, m:=\left\langle\widetilde{\nabla}_{X_{y}} X_{x}, N\right\rangle$ are given by

$$
l=\frac{-u_{y} / y+u_{x x}}{\sqrt{1+y^{2}\left(u_{x}^{2}+u_{y}^{2}\right)}}, \quad n=\frac{u_{y} / y+u_{y y}}{\sqrt{1+y^{2}\left(u_{x}^{2}+u_{y}^{2}\right)}}, \quad m=\frac{u_{x} / y+u_{x y}}{\sqrt{1+y^{2}\left(u_{x}^{2}+u_{y}^{2}\right)}} .
$$

Now the Gram-Schmidt orthogonalization process provides a tangent field $Y=X_{y}$ $-\left\langle X_{y}, X_{x}\right\rangle X_{x} /\left\|X_{x}\right\|^{2}$ orthogonal to $X_{x}$. Let $\overrightarrow{\boldsymbol{H}}$ be the mean curvature vector. Now taking into account the fact that the mean curvature $H$ defined by $\overrightarrow{\boldsymbol{H}}=H N$ is given by $2 H=\left(1 /\left\|X_{x}\right\|^{2}\right)\left\langle\widetilde{\nabla}_{X_{x}} X_{x}, N\right\rangle+\left(1 /\|Y\|^{2}\right)\left\langle\widetilde{\nabla}_{Y} Y, N\right\rangle$, we infer the formula for the vertical mean curvature equation in our model stated in the introduction, see Equation (4). We now observe that this equation is of divergence form, given by Equation (5).

We observe that any vertical minimal graph or $H$-graph is stable. In fact, vertical translations provide a foliation of an open subset of ambient space given by $H$-surfaces, transverse to the Killing vertical vector field; see [15].

In particular, we now focus on vertical graphs of the form $u(x, y)=\lambda(y)+\ell x$; we say that $t=\lambda(y)$ is the generating curve. In view of (4), we have therefore that the generating curve $t=\lambda(y)$ of a parabolic screw motion surface satisfies the following equation.

PROPOSITION 2.1 (Mean curvature equation). The mean curvature equation is

$$
\frac{2 H}{y^{2}}\left(1+y^{2}\left(\ell^{2}+\lambda^{\prime 2}\right)\right)^{3 / 2}=\left(1+y^{2} \ell^{2}\right) \lambda^{\prime \prime}-y \lambda^{\prime}\left(\ell^{2}+\lambda^{\prime 2}\right) .
$$

Owing to the fact that the mean curvature Equation (4) is of divergence form (5), we derive the following crucial formula for parabolic screw motion surfaces. 
LEMMA 2.1 (First integral).

$$
\left(\frac{\lambda^{\prime}}{\sqrt{1+y^{2}\left(\ell^{2}+\lambda^{\prime 2}\right)}}\right)^{\prime}=\frac{2 H(y)}{y^{2}} .
$$

In particular, if $H$ is constant, we obtain

$$
\left.\frac{\lambda^{\prime}}{\sqrt{1+y^{2}\left(\ell^{2}+\lambda^{\prime 2}\right)}}=\frac{y d-2 H}{y} \quad \text { (first integral }\right) .
$$

We remark that Equations (6) and (8) can be alternatively inferred using the techniques derived in [19].

Finally, we observe that in our model the horizontal mean curvature Equation (2), satisfied by a positive smooth function $y=g(x, t)$, can also be inferred in a similar way. For the readers benefit, we now give the principal quantities that arise in the derivation of this formula: the unit normal $N$ pointing towards the half-space $\{y>0\}$ is given by

$$
N=\frac{1}{\sqrt{g_{t}^{2}+g^{2}\left(g_{x}^{2}+1\right)}}\left(-g_{x} g^{2}, g^{2},-g_{t}\right) .
$$

The coefficients of the second fundamental form are given (in the same notation as before) by

$$
\begin{gathered}
l=\frac{g_{x x}+(1 / g)+\left(g_{x}^{2} / g\right)}{\sqrt{g_{t}^{2}+g^{2}\left(g_{x}^{2}+1\right)}}, \\
n=\frac{g_{t t}-\left(g_{t}^{2} / g\right)}{\sqrt{g_{t}^{2}+g^{2}\left(g_{x}^{2}+1\right)}}, \quad m=\frac{g_{x t}}{\sqrt{g_{t}^{2}+g^{2}\left(g_{x}^{2}+1\right)}} .
\end{gathered}
$$

We will see in the next section how these graphs arise in the construction of parabolic screw motion surfaces.

2.1. Complete embedded minimal surfaces We will now state the following existence theorem for minimal surfaces in $\mathbb{H}^{2} \times \mathbb{R}$ obtained by the construction of complete horizontal graphs in our model.

THEOREM 2.1 (Existence of embedded minimal surfaces).

(a) The tilted Euclidean half-plane

$$
t=\ell x, \quad y>0
$$

gives rise to a one-parameter family of complete embedded simply connected minimal stable surfaces in $\mathbb{H}^{2} \times \mathbb{R}$ invariant by parabolic screw motions. Two such surfaces with different pitches $\ell$ are nonisometric. If $\ell=0$ we get the hyperbolic plane. 
(b) Let us assume that $d \neq 0$. The generating curve is obtained by gluing together a convex vertical graph $t=\lambda(y), 0 \leqslant y \leqslant 1 / d$, vertical at $y=1 / d$, and its vertical reflection. We obtain a horizontal graph family $y=g_{H}(x, t, d, \ell)$, $d \neq 0$, of complete embedded simply connected minimal surfaces invariant by parabolic screw motions. Fixing the parameter $d$ and varying $\ell$, we get a family of nonisometric deformations of Daniel-Hauswirth minimal surface, that is, two surfaces with different pitches $\ell$ are nonisometric.

We will see in Section 5 that, taking $\ell=1$ in (9), we obtain a conjugate Scherk-type minimal surface.

PROOF. Now observe that it follows from (8) that $\lambda^{\prime} \equiv 0$ produces a solution; hence, up to vertical translation or symmetry about the $x y$ plane, we may assume that $\lambda \equiv 0$. Now, on account of (1), we deduce that the tilted Euclidean half-plane $t=\ell x, y>0$ gives rise to a one-parameter family of complete embedded minimal stable surfaces in $\mathbb{H}^{2} \times \mathbb{R}$ invariant by screw motions. On account of Corollary 4.1, Equation (35) (taking $H=0$ ), we see that two such immersions with different pitches $\ell$ are nonisometric. If $\ell=0$ we get the hyperbolic plane. Now let us suppose that $d \neq 0$. From (8), up to vertical translation or symmetry about the $x y$ plane, we have that the minimal parabolic screw motion vertical graph $t=\lambda(y)+\ell x$, generated by $t=\lambda(y)=\lambda(y, d, \ell), y \leqslant 1 / d$, is given by

$$
t=d \int_{0}^{y} \frac{\sqrt{1+\xi^{2} \ell^{2}}}{\sqrt{1-\xi^{2} d^{2}}} d \xi .
$$

Let EllipticE $(k)$ be the complete elliptic integral of the second kind. Note now that we get an incomplete elliptic integral of the second kind in (10), since $\lambda(y, d, \ell)=\operatorname{EllipticE}\left(y d, \sqrt{(-1) \ell^{2} / d^{2}}\right)$. Now it is a simple calculation to deduce that $t=\lambda(y)$, defined by (10), is increasing and convex in the interval $[0,1 / d)$, strictly convex in the interval $(0,1 / d)$, and it is vertical at the point $y=1 / d$. Another computation shows that the Euclidean curvature at $y=1 / d$ is finite and that $\lambda(1 / d)=\operatorname{EllipticE}\left(\sqrt{-\ell^{2} / d^{2}}\right)$. Hence, by gluing together $t=\lambda(y)$ with its vertical reflection, say the Schwarz reflection, given by $2 \operatorname{EllipticE}\left(\sqrt{-\ell^{2} / d^{2}}\right)-\lambda(y)$, we obtain an embedded curve which is complete in the ambient space and has 'sinusoidal shape' (from the Euclidean view point). Now, in view of (1), by applying successive screw motions to this curve, which is a Euclidean translation in the direction of the vector $(1,0, \ell)$, we get the horizontal minimal graph $y=g_{H}(x, t, d, \ell)$, $d \neq 0$. More precisely, this horizontal minimal graph is obtained by gluing together the vertical graph $t=\lambda(y, d, \ell)+\ell x, y \leqslant 1 / d$, with its Schwarz reflection given by 2EllipticE $\left(\sqrt{-\ell^{2} / d^{2}}\right)-\lambda(y, d, \ell)+\ell x$. Of course, this construction yields a complete embedded simply connected minimal surface invariant by parabolic screw motions. Now, by fixing the parameter $d$ and letting $\ell$ vary, we get from Corollary 4.1, Equation (36) (making $H=0$ ), a nonisometric deformation of the Daniel-Hauswirth minimal surface $(\ell=0)$. This completes the proof of the theorem, as desired. 
REMARK 1. (1) Let $t=\lambda(y)=\lambda(y, d, \ell), y \leqslant 1 / d, d \neq 0$ be a generating curve of an embedded minimal surface given by Theorem 2.1 . Recall that by gluing together $t=\lambda(y)$ with its Schwarz reflection, given by $2 \operatorname{EllipticE}\left(\sqrt{-\ell^{2} / d^{2}}\right)-\lambda(y)$, we obtain an embedded curve generating an embedded minimal surface. Now, for $\zeta>0$, let us define $f(y):=\lambda(\zeta y), y \leqslant 1 /(\zeta d)$. It is easy to verify that $f(y)$ satisfies the minimal Equation (8) $(H=0)$, with pitch $\zeta \ell$ and parameter $\zeta d$. Now we observe that the resulting minimal surface can be obtained geometrically, by applying a horizontal translation (horizontal homothety) $(x, y, \lambda(y)+\ell x) \rightarrow(\zeta x, \zeta y, \lambda(y)+\ell x)$ to the original surface. Thus, making $d=\ell$, we obtain a foliation of an open set of the $y t$ vertical plane $(y>0)$ given by a one-parameter family of embedded complete curves generating a one-parameter family of embedded minimal surfaces. Some of these generating curves are drawn in Figure $2(d=\ell=4,2,1,2 / 3,1 / 2)$.

(2) Now let us fix $\ell$, say $\ell=1$, for simplicity. We will see that, by varying $d$ in the interval $(0, \infty)$, we obtain a foliation of the half $y t$-vertical plane $(y>0, t>0)$ by generating curves of embedded, minimal, parabolic screw motion surfaces, producing a foliation of an open set of ambient space. Consider positive numbers $d_{1}, d_{2}$ with $d_{2}<d_{1}$. Notice that $\lambda\left(y, d_{1}, 1\right)>\lambda\left(y, d_{2}, 1\right)$ if $y \leqslant 1 / d_{1}$. Now observe also that $\lambda(1 / d)=\operatorname{EllipticE}\left(\sqrt{-1 / d^{2}}\right)$ is a strictly decreasing function in the variable $d$, satisfying $\lambda(1 / d) \rightarrow \infty$, as $d \rightarrow 0$, and $\lambda(1 / d) \rightarrow \pi / 2$, as $d \rightarrow \infty$. Hence, we deduce that for $d_{2}<d_{1}$ the generating curve obtained by $\lambda\left(y, d_{2}, 1\right)$ and its Schwarz reflection 'involves' (in the Euclidean sense) entirely the curve determined by $\lambda\left(y, d_{1}, 1\right)$ and its Schwarz reflection, see Figure 3. Consequently, we infer the desired foliation of the half-vertical plane. Hence, we get a foliation of an open set of ambient space by minimal parabolic screw motion surfaces with pitch $\ell=1$. In Figure 3 some generating curves for $\ell=1$ and $d=1 / 2,2 / 3,1,3 / 2,2,10$ are drawn.

(3) Finally, we see that $t=\lambda(y, 1, \ell), y \leqslant 1$ gives the generating curve of a family of nonisometric deformations of the Daniel-Hauswirth minimal surface, obtained by fixing the parameter $d=1$ and varying the pitch $\ell$. We obtain an embedded minimal surface by gluing together $t=\lambda(y, 1, \ell)+\ell x, y \leqslant 1$ and its Schwarz reflection, as we have explained before. Notice that this family of generating curves has selfintersections. In Figure 4 we show some examples for $\ell=1 / 100,1,2,3$. We leave the description of their asymptotic boundaries to the reader.

2.2. Complete embedded $\boldsymbol{H}$-surfaces We will prove our existence results for parabolic screw motion $H$-surfaces in $\mathbb{H}^{2} \times \mathbb{R}$, if the mean curvature satisfies $|H|<1 / 2$. We will construct complete $H$-surfaces given by vertical graphs over the entire hyperbolic plane; hence they are stable. Whether there exists a noncompact complete stable $H$-surface in $\mathbb{H}^{2} \times \mathbb{R}$ with mean curvature bigger than $1 / 2$ is an interesting question. This is related to results derived by Nelli and Rosenberg. These authors proved that in $\mathbb{H}^{2} \times \mathbb{R}$ there is no noncompact complete stable $H$-surface with $H>1 / \sqrt{3}$, either with or without compact boundary [15]. Does there exist a complete noncompact stable $H$-surface with $H$ satisfying $1 / 2<H \leqslant 1 / \sqrt{3}$ ? For $H=1 / 2$, surfaces of revolution provide examples of complete noncompact stable $H$-surfaces, which have a simple explicit expression, see [1] or [15]. See also [19]. 


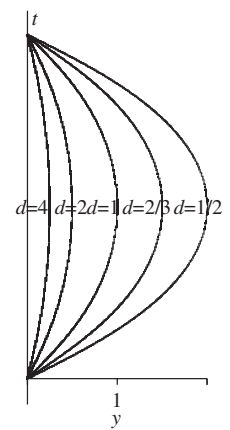

FIGURE 2. Generating curves of embedded minimal surfaces obtained by horizontal translations varying the pitch $\ell=d$.

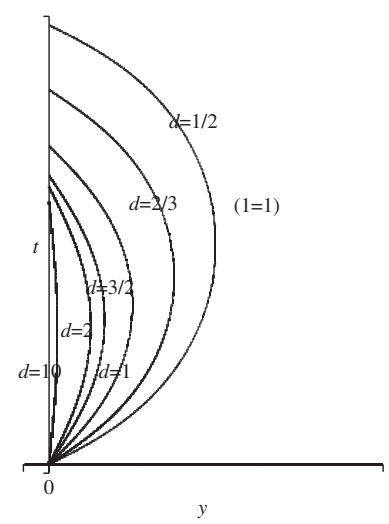

FIGURE 3. Foliation given by generating curves of embedded minimal surfaces obtained by varying $d$ and fixing the pitch $\ell=1$.

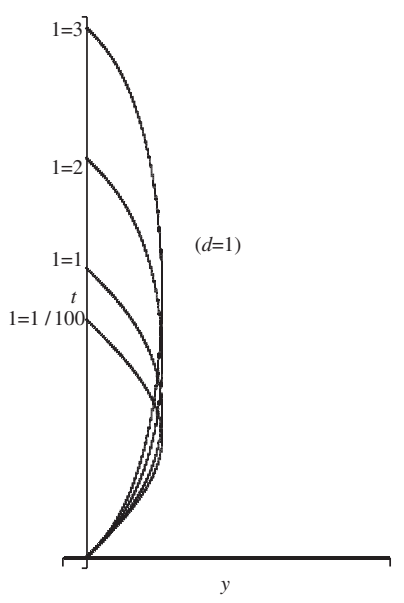

FIGURE 4. Generating curves of embedded minimal surfaces obtained by varying the pitch $\ell$ and fixing the parameter $d=1$. 

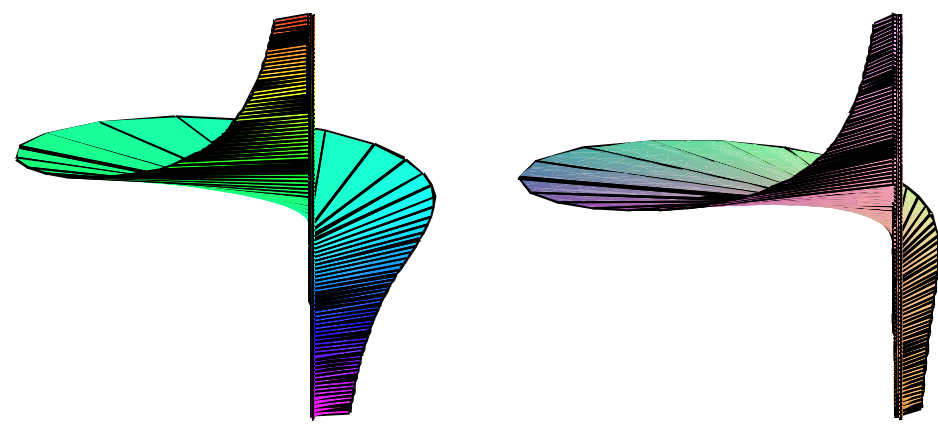

FIgURE 5. The ball model for $\mathbb{H}^{2} \times\{0\}$. Nonisometric deformation in $\mathbb{H}^{2} \times \mathbb{R}$ of the hyperbolic plane $(\ell=1)$. A conjugate Scherk-type minimal surface.
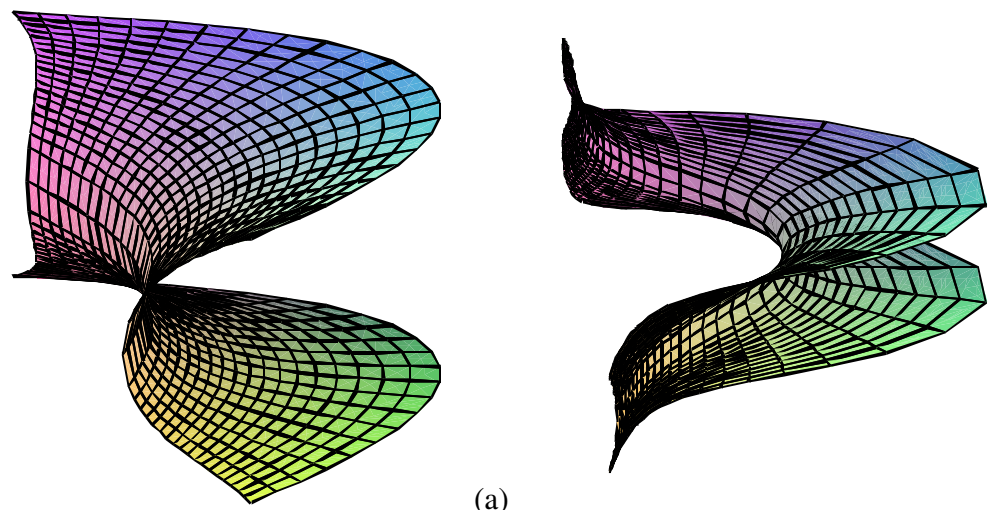

(a)

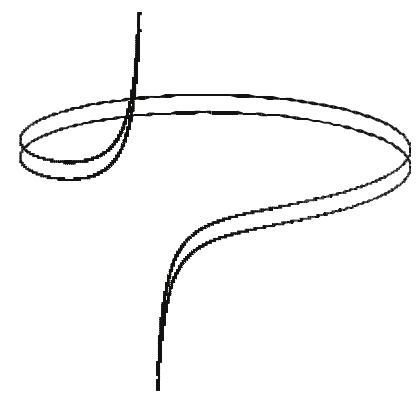

(b)

FIgurE 6. The ball model for $\mathbb{H}^{2} \times\{0\}$. (a) Nonisometric deformation $(d=\ell=1)$ in $\mathbb{H}^{2} \times \mathbb{R}$ of the Daniel-Hauswirth minimal surface. (b) The asymptotic boundary of a nonisometric deformation of the Daniel-Hauswirth minimal surface $(d=\ell=1)$. 
We will obtain in the next section examples of entire vertical graphs with mean curvature $H=1 / 2$.

TheOREM 2.2 (Existence of embedded $H$-surfaces). For any $H$ in $(-1 / 2,0)$, there exists a one-parameter explicit family of vertical $H$-graphs with pitch $\ell$, each one if which is stable and embedded, given by $t=u_{\mathcal{H}}(x, y, \ell):=\lambda(y, \ell)+\ell x$ over the entire hyperbolic plane $\mathbb{H}^{2} \times\{0\}$. Varying $\ell$, we obtain a family of nonisometric, simply connected and embedded, stable deformations of the Abresch-Rosenberg surface $(\ell=0)$. More precisely, the generating curve $t=\lambda(y, \ell)$ is given by

$$
t=\lambda(y, \ell)=\frac{-2 H}{\sqrt{1-4 H^{2}}}\left[\ln \left(\frac{\sqrt{1+\ell^{2} y^{2}}-1}{\sqrt{1+\ell^{2} y^{2}}+1}\right)^{1 / 2}+\sqrt{1+\ell^{2} y^{2}}\right] \quad(\ell \neq 0) .
$$

For any $H$ in $(-1 / 2,0)$, there exists a two-parameter explicit family of horizontal $H$-graphs given by $y=g_{\mathcal{H}}(x, t, d, \ell)$ over the entire $x t$ plane, each one of which is embedded, that contains the above family: if $d=0$, the generating curve is given by (11). These horizontal H-graphs are obtained by applying Schwarz reflection to a vertical parabolic screw motion graph generated by $t=\lambda(y, d, \ell), 0<y<(1-2|H|) / d$, and its vertical reflection at the vertical point $y=(1-2|H|) / d$. Fixing $d$ and letting $\ell$ vary, we obtain a family of nonisometric deformations. Thus, each such $H$-surface is a complete simply connected embedded surface, invariant by parabolic screw motions.

PROOF. From (8), up to vertical translation or symmetry about the $x y$ plane, we have that a screw motion $H$-vertical graph $t=\lambda(y, d, \ell)+\ell x$, with $|H|<1 / 2$, generated by $t=\lambda(y, d, \ell)$, is given by

$$
t=\int_{*}^{y} \frac{(2|H|+\xi d) \sqrt{1+\xi^{2} \ell^{2}}}{\xi \sqrt{1-(2|H|+\xi d)^{2}}} d \xi \quad(d \geqslant 0) .
$$

Letting $d=0$ in (12), we infer that

$$
t:=\lambda(y, \ell)=\frac{2|H|}{\sqrt{1-4 H^{2}}} \int_{*}^{y} \frac{\sqrt{1+\xi^{2} \ell^{2}}}{\xi} d \xi .
$$

The behavior of $\lambda(y, \ell)$ can be analyzed as follows. Notice that on account of (8) we are assuming that $|H|<1 / 2$, with $H<0$. Now clearly $\lambda(y, \ell)$ is an increasing function for $y>0$. A computation shows that $\lambda(y, \ell)$ is strictly concave. Next, notice that $\sqrt{1+y^{2} \ell^{2}}=1+\left(\ell^{2} / 2\right) y^{2}+o\left(y^{2}\right)$, near $y=0$; hence $\lambda(y, \ell)$ has a log behavior as $y \rightarrow 0$. Clearly, at infinity $\lambda(y, \ell)$ has a linear behavior. Thus, $u_{\mathcal{H}}(x, y, \ell)$ $:=\lambda(y, \ell)+\ell x$ is a vertical graph over the entire hyperbolic plane $\mathbb{H}^{2} \times\{0\}$, which yields a complete horizontal $H$-graph over the $(x, t)$ plane, as well. We conclude therefore that there exists a one-parameter family of vertical $H$-graphs, and each one is stable and embedded. On the other hand, observe that Equation (13) is easily solved by elementary integration techniques: we therefore obtain the explicit form (11) in the theorem, the graph of which, for $H=-1 / 4, \ell=1$, is drawn in Figure 7. Now, if $d \neq 0$, we define $\lambda(y d, \ell)$ by (12). Clearly, $\lambda(y, d, \ell)$ is an 


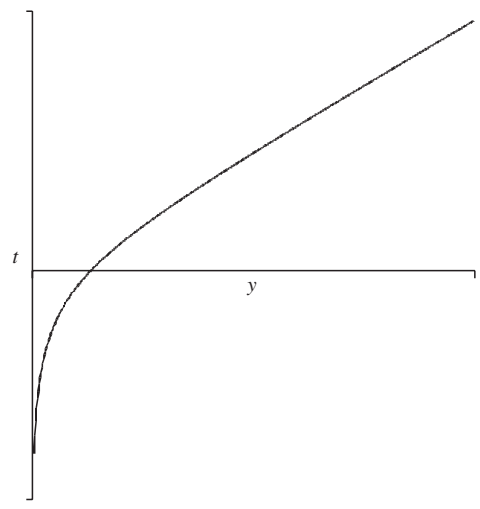

FIGURE 7. The generating curve of an embedded $H$-surface belonging to the family $(d=0)$ given by the elementary formula (11).

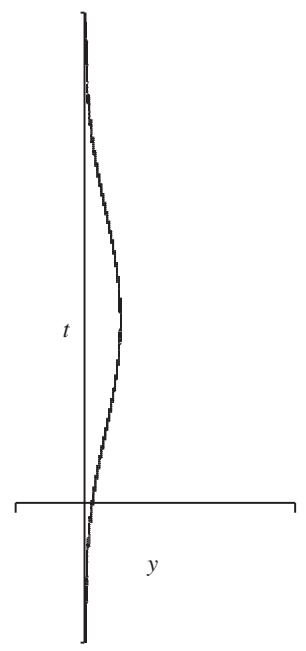

FIGURE 8. The generating curve of an embedded $H$-surface $(d=\ell=1)$.

increasing function, which is vertical at $y=(1-2|H|) / d$. By reasoning analogous to that used above, the local behavior of $\lambda(y, d, \ell)$ at $y=0$ is of log type. Now after a computation we infer that at $y=(1-2|H|) / d$ the Euclidean curvature is finite, so hence by vertical reflection at $y=(1-2|H|) / d$ we obtain a complete horizontal $H$-graph $y=g_{H}(x, t, d, \ell)(|H|<1 / 2)$ over the entire $(x, t)$ plane, which is simply connected, embedded and invariant by parabolic screw motions. The generating curve for $H=-1 / 4, d=\ell=1$, is drawn in Figure 8. It follows from Corollary 4.1 that, by fixing $d$ and varying $\ell$, the family $t=u_{H}(x, y, \ell)(d=0)$ is determined by (11) and the family $y=g_{H}(x, t, d, \ell)$ is formed by nonisometric $H$-surfaces. This concludes the proof of the theorem, as desired. 


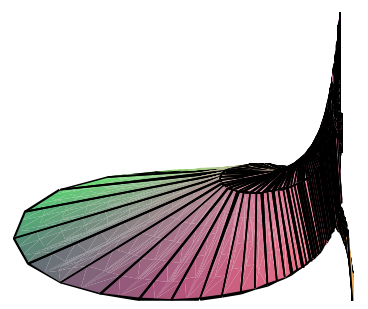

FIgURE 9. The ball model for $\mathbb{H}^{2} \times\{0\}$. A nonisometric, simply connected and embedded stable deformation $(d=0, \ell=1)$ in $\mathbb{H}^{2} \times \mathbb{R}$ of the Abresch-Rosenberg surface, $H=-1 / 4$.

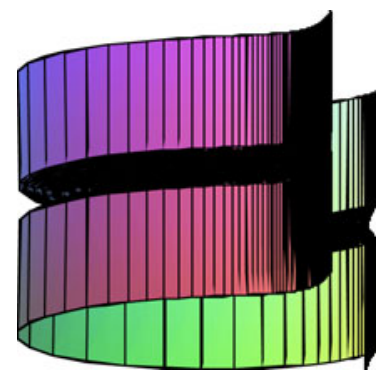

FIgURE 10. The ball model for $\mathbb{H}^{2} \times\{0\}$. A complete embedded $H$-surface $(d=1, \ell=1)$ in $\mathbb{H}^{2} \times \mathbb{R}$, invariant by parabolic screw motions, $H=-1 / 4$.

\section{A generalized Bour lemma}

We will now prove a generalized Bour lemma that can be applied to parabolic and hyperbolic screw motion surfaces in $\mathbb{H}^{2} \times \mathbb{R}$ and screw motion surfaces in Heisenberg's space. This generalizes a result in [19]. Ordóñes, following ideas of Do Carmo and Dajczer [4], proved another generalization of the Bour lemma applied to surfaces invariant by screw motions in a three-dimensional space form. It is reasonable to expect a general Bour lemma that holds in all situations. However, we will not pursue this task here.

We will now consider the three-dimensional Riemannian manifold $M^{3}$ given by an open set of the Euclidean 3 -space $\mathbb{R}^{3}$ equipped with coordinates $\{(\rho, \varphi, t), \rho>0\}$ and metric $d \sigma^{2}$ given by

$$
d \sigma^{2}=\Psi^{2}(\rho) d \rho^{2}+\Phi^{2}(\rho) d \varphi^{2}-\Lambda^{2}(\rho) d t d \varphi+d t^{2}
$$

where $\Psi^{2}, \Phi^{2}$ and $\Lambda^{2}$ satisfy some further conditions, as we will see in what follows. We will call $\Gamma$ the one-parameter group of isometries acting on $M^{3}$ by translation on the $\varphi$ variable.

Definition 3.1. We say that a surface $S$ immersed into $M^{3}$ is a $\Gamma$-screw motion surface (with vertical axis $t$ ) if it is invariant by successive compositions of an element 
of $\Gamma$ with a vertical translation. More precisely, using coordinates $(\rho, \varphi, t), S$ is given by $(\rho, \varphi) \mapsto(\rho, \varphi, \lambda(\rho)+\ell \varphi)$, where $\ell \geqslant 0$, is called the pitch. We say that the curve $(\rho, \lambda(\rho))$ lying on $\{\varphi=0\}$ is the generating curve.

We have studied in [19] the generating curve of standard screw motion surfaces in $\mathbb{H}^{2} \times \mathbb{R}$ and $\mathbb{S}^{2} \times \mathbb{R}$. In Section 2 , we studied the generating curve of parabolic screw motion surfaces in $\mathbb{H}^{2} \times \mathbb{R}$.

Next, let us give some motivating examples to see that the metric given by (14) appears naturally in this context.

\section{REMARK 2.}

(1) The ambient space is $\mathbb{H}^{2} \times \mathbb{R}$.

(a) Standard screw motion surfaces. Here we take the ball model $\mathbb{H}^{2}=$ $\left\{(x, y), x^{2}+y^{2}<1\right\}$ as the hyperbolic plane equipped with the hyperbolic metric $\left(4 /\left(1-|z|^{2}\right)^{2}\right)|d z|^{2}$. The metric in $\mathbb{H}^{2} \times \mathbb{R}$ using cylindrical coordinates $(\rho, \varphi, t)$ (where $\rho$ is the hyperbolic distance measure from the origin of $\mathbb{H}^{2}$, that is, $R=$ $\tanh \rho / 2, R=\sqrt{x^{2}+y^{2}}$ and $t$ is the height) is given by $d \sigma^{2}=d \rho^{2}+\sinh ^{2} \rho d \varphi^{2}+$ $d t^{2}$. Thus we have $\Phi^{2}=\sinh ^{2} \rho, \Phi^{\prime 2}=\cosh ^{2} \rho=F_{1}\left(\Phi^{2}\right)$, where $F_{1}(u)=1+u$. $\Psi^{2} \equiv 1$ and $\Lambda \equiv 0$.

(b) Parabolic screw motion surfaces. We take the upper half-plane model $\mathbb{H}^{2}=$ $\{(x, y), y>0\}$ equipped with the hyperbolic metric $\left(d x^{2} / y^{2}\right)+\left(d y^{2} / y^{2}\right)$. We set $y=\rho$ and $x=\varphi$. The metric is given by $d \sigma^{2}=\left(d x^{2} / y^{2}\right)+\left(d y^{2} / y^{2}\right)+d t^{2}$. We have $\Phi^{2}=1 / y^{2}, \Phi^{\prime 2}=1 / y^{4}=F_{1}\left(\Phi^{2}\right)$, where $F_{1}(u)=u^{2} . \Psi^{2}=G_{1}\left(\Phi^{2}\right)$, where $G_{1}(u)=u$ and $\Lambda \equiv 0$.

(c) Hyperbolic screw motion surfaces. We again take the upper half-plane model and polar coordinates $x=R \cos \theta, y=R \sin \theta, 0<\theta<\pi, R>0$. In view of (14), we set $\rho:=\theta$ and $e^{\varphi}:=R$. Thus we have $\mathbb{H}^{2}=\left\{\left(x=e^{\varphi} \cos \rho, y=e^{\varphi} \sin \rho\right)\right.$, $0<\rho<\pi\}$, equipped with the hyperbolic metric. The metric in $\mathbb{H}^{2} \times \mathbb{R}$ using coordinates $(\rho, \varphi, t)$ is given by $d \sigma^{2}=\left(d \rho^{2} / \sin ^{2} \rho\right)+\left(d \varphi^{2} / \sin ^{2} \rho\right)+d t^{2}$. We have $\Phi^{2}=1 / \sin ^{2} \rho, \Phi^{\prime 2}=F_{1}\left(\Phi^{2}\right)$, where $F_{1}(u)=u^{2}-u . \Psi^{2}=G_{1}\left(\Phi^{2}\right)$, where $G_{1}(u)=u$ and $\Lambda \equiv 0$.

(2) Screw motion surfaces in $\mathbb{S}^{2} \times \mathbb{R}$. Now let $\mathbb{S}^{2}=\mathbb{C} \cup\{\infty\}$ be the sphere equipped with the spherical metric $\left(4 /\left(1+|z|^{2}\right)^{2}\right)|d z|^{2}$. The metric in $\mathbb{S}^{2} \times \mathbb{R}$ using cylindrical coordinates $(\rho, \varphi, t)$ (where $\rho$ is the sphere distance measure from the origin of $\mathbb{S}^{2}$, that is, $R=\tan \rho / 2, R=\sqrt{x^{2}+y^{2}}$ and $t$ is the height) is given by $d s^{2}=$ $d \rho^{2}+\sin ^{2} \rho d \varphi^{2}+d t^{2}$. We have $\Phi^{2}=\sin ^{2} \rho, \Phi^{\prime 2}=F_{1}\left(\Phi^{2}\right)$, where $F_{1}(u)=1-u$. $\Psi^{2} \equiv 1$ and $\Lambda \equiv 0$.

(3) Screw motion surfaces in Heisenberg space. Consider $\mathbb{R}^{3}$ equipped with the metric $d \sigma^{2}=d x^{2}+d y^{2}+[d t+(1 / 2)(y d x-x d y)]^{2}$. We write $x=$ $2 \sinh (\rho / 2) \cos \varphi, y=2 \sinh (\rho / 2) \sin \varphi$. The metric using coordinates $(\rho, \varphi, t)$ is given by

$$
d \sigma^{2}=\cosh ^{2}(\rho / 2) d \rho^{2}+\sinh ^{2} \rho d \varphi^{2}-4 \sinh ^{2}(\rho / 2) d t d \varphi+d t^{2} .
$$


We have $\Phi^{2}=\sinh ^{2} \rho, \Phi^{\prime 2}=F_{1}\left(\Phi^{2}\right)$, where $F_{1}(u)=1+u . \Psi^{2}=G_{1}\left(\Phi^{2}\right)$, where $G_{1}(u)=(1+\sqrt{1+u}) / 2$. Also $\Lambda^{2}=G_{2}\left(\Phi^{2}\right)$, where $G_{2}(u)=2(\sqrt{1+u}-1)$, $\Lambda^{\prime 2}=G_{1}\left(\Phi^{2}\right)$.

Next, we will prove a central result in this section. It contains some apparently complicated formulas but, as we will see afterwards, when it is specialized to parabolic or hyperbolic screw motion surfaces the formulas look much nicer.

THEOREM 3.1 (Generalized Bour lemma). Let $M^{3}$ be the Euclidean 3-space endowed with the metric given by (14) and the one-parameter group of isometries $\Gamma$. Let us assume that $\Phi^{2}+\ell^{2}>\ell \Lambda^{2}$ and $\Phi^{2}>\Lambda^{4} / 4$. Then, any surface invariant by $\Gamma$-screw motions can be parameterized locally by natural coordinates $s, \tau$, such that the induced metric $d \mu^{2}$ is given by

$$
d \mu^{2}=d s^{2}+U^{2}(s) d \tau^{2} .
$$

Furthermore, assume that $\Phi^{2}=f\left(U^{2}\right), \Phi^{\prime 2}=F_{1}\left(\Phi^{2}\right), \Psi^{2}=G_{1}\left(\Phi^{2}\right), \Lambda^{2}=G_{2}\left(\Phi^{2}\right)$ and $\Lambda^{\prime 2}=G_{3}\left(\Phi^{2}\right)$, where $f(u), F_{1}(u), G_{1}(u), G_{2}(u)$ and $G_{3}(u)$ are smooth real functions for $u \geqslant 0$. Let $S$ be such a $\Gamma$-screw motion surface. Then there exists a twoparameter family $\mathcal{F}(m, \ell), m \neq 0$ of $\Gamma$-screw motion surfaces isometric to $S$, given by

$$
\begin{gathered}
m^{2} U^{2}=\ell^{2}+\Phi^{2}-\Lambda^{2} \ell, \\
\rho^{\prime 2}(s)=\frac{\ell^{2}+\Phi^{2}-\Lambda^{2} \ell}{\Psi^{2}\left(\ell^{2}+\Phi^{2}-\Lambda^{2} \ell\right)+\lambda^{\prime 2}(\rho)\left(\Phi^{2}-\Lambda^{4} / 4\right)}, \\
\rho^{\prime 2}(s)=\frac{m^{4} U^{2} U^{\prime 2}}{\left[\sqrt{f\left(U^{2}\right)} \sqrt{F_{1}\left(f\left(U^{2}\right)\right)}-\ell \sqrt{G_{2}\left(f\left(U^{2}\right)\right) G_{3}\left(f\left(U^{2}\right)\right)}\right]^{2}}, \\
\lambda \circ \rho(s)=\int \frac{m U}{\left[m^{2} U^{2}-\left(\ell-G_{2}\left(f\left(U^{2}\right)\right) / 2\right)^{2}\right]^{1 / 2}} \cdot B d s,
\end{gathered}
$$

where

$$
\begin{gathered}
B(s)=\left[1-\frac{m^{4} U^{2} U^{\prime 2} G_{1}\left(f\left(U^{2}\right)\right)}{\left[\sqrt{f\left(U^{2}\right) F_{1}\left(f\left(U^{2}\right)\right)}-\ell \sqrt{G_{2}\left(f\left(U^{2}\right)\right) G_{3}\left(f\left(U^{2}\right)\right)}\right]^{2}}\right]^{1 / 2}, \\
\varphi(s, \tau)=\frac{\tau}{m}-\int \frac{(\lambda \circ \rho)^{\prime}\left[\ell-G_{2}\left(f\left(U^{2}\right)\right) / 2\right]}{m^{2} U^{2}} d s .
\end{gathered}
$$

PROOF. The proof will proceed in the same sprit as in [19]. In view of (14) and $t=\lambda(\rho)+\ell \varphi$, we deduce that the induced metric $d \mu^{2}$ of a given screw motion $\Gamma$-surface $S$ immersed into $M^{3}$ is given by

$$
d \mu^{2}=\left(\Psi^{2}+\lambda^{\prime 2}\right) d \rho^{2}+\left(\Phi^{2}+\ell^{2}-\Lambda^{2} \ell\right) d \varphi^{2}+\left(2 \ell \lambda^{\prime}-\Lambda^{2} \lambda^{\prime}\right) d \rho d \varphi
$$


Now rewrite the above equation to obtain

$$
\begin{aligned}
d \mu^{2}= & \underbrace{\left[\Psi^{2}+\frac{\lambda^{\prime 2}\left(\Phi^{2}-\Lambda^{4} / 4\right)}{\ell^{2}+\Phi^{2}-\Lambda^{2} \ell}\right] d \rho^{2}}_{d s^{2}} \\
& +\underbrace{\left[\ell^{2}+\Phi^{2}-\Lambda^{2} \ell\right]}_{U^{2}} \underbrace{\left[d \varphi+\frac{\left(\ell-\Lambda^{2} / 2\right)}{\ell^{2}+\Phi^{2}-\Lambda^{2} \ell} d \lambda \circ \rho\right]^{2}}_{d \tau^{2}} .
\end{aligned}
$$

This equation leads to the following system:

$$
\begin{aligned}
& d s=\sqrt{\Psi^{2}+\frac{\lambda^{\prime 2}\left(\Phi^{2}-\Lambda^{4} / 4\right)}{\ell^{2}+\Phi^{2}-\Lambda^{2} \ell^{2}}} d \rho, \\
& d \tau=d \varphi+\frac{\lambda^{\prime}\left(\ell-\Lambda^{2} / 2\right)}{\ell^{2}+\Phi^{2}-\Lambda^{2} \ell} d \rho .
\end{aligned}
$$

By the implicit function theorem (due to the fact that $\partial(s, \tau) / \partial(\rho, \varphi) \neq 0$ ), we define locally natural coordinates $s, \tau$. Notice that $\rho$ and $\lambda$ do not depend on $\tau$; hence $U=U(s)$ and we deduce (15). Next we search for an explicit parametrization of an arbitrary screw motion surface (with pitch denoted by $\ell$ for convenience) isometric to $S$ by natural coordinates $s, \tau$, involving a simple expression in terms of $U$ and parameters $\ell, m$ as in the theorem. Notice that we may suppose that $U>0$, since we assume momentarily that $\rho>0$ (an extension to $\rho=0$ or negative values requires some additional argument). In view of (19) we infer the third equation in the theorem; hence

$$
(\lambda \circ \rho)^{\prime 2}=\left(\frac{\ell^{2}+\Phi^{2}-\Lambda^{2} \ell}{\Phi^{2}-\Lambda^{4} / 4}\right)\left(1-\rho^{\prime 2} \Psi^{2}\right) .
$$

Now on account of (18) we see that the expression of $U^{2}$ is given by

$$
U d \tau= \pm \sqrt{\ell^{2}+\Phi^{2}-\Lambda^{2} \ell}\left[d \varphi+\frac{\lambda^{\prime}\left(\ell-\Lambda^{2} / 2\right)}{\ell^{2}+\Phi^{2}-\Lambda^{2} \ell} d \rho\right] .
$$

Thus

$$
\begin{aligned}
& \frac{\partial \varphi}{\partial \tau}= \pm \frac{U}{\sqrt{\ell^{2}+\Phi^{2}-\Lambda^{2} \ell}}, \\
& \frac{\partial \varphi}{\partial s}=\frac{-\lambda^{\prime} \rho^{\prime}\left(\ell-\Lambda^{2} / 2\right)}{\ell^{2}+\Phi^{2}-\Lambda^{2} \ell} .
\end{aligned}
$$

In view of (22) and (23), we deduce that $\partial \varphi / \partial \tau$ does not depend on $s$; hence we obtain the crucial formula

$$
\pm \frac{U}{\sqrt{\ell^{2}+\Phi^{2}-\Lambda^{2} \ell}}=\frac{1}{m}, \quad m \neq 0 .
$$


Then we derive the second formula in the theorem. From (24), by performing some calculations we are able to express $\rho^{\prime 2}$ in terms of $U^{2}, U^{\prime 2}, m^{4}$ and $\left(\Phi \Phi^{\prime}-\Lambda \Lambda^{\prime} \ell\right)^{2}$. Again taking into account (24), by substituting $\Phi, \Phi^{\prime}, \Lambda$ and $\Lambda^{\prime}$ in terms of $\Phi^{2}=$ $f\left(U^{2}\right)$, in view of (21), we derive the fourth and fifth formulas in the theorem. The last formula follows by combining (22), (23) and (24) with the fifth formula. This concludes the proof of the second part and therefore the proof of the theorem.

REMARK 3. We note that from Theorem 3.1 we recover the formulas given in [19], Theorem 19 and Theorem 20, for screw motion surfaces in $\mathbb{H}^{2} \times \mathbb{R}$ and $\mathbb{S}^{2} \times \mathbb{R}$, see Remark 2, examples (1)(a) and (2) above. In fact, when the ambient space is the product $M^{2} \times \mathbb{R}$, we have $\Lambda \equiv 0\left(G_{2}=G_{3} \equiv 0\right)$. Moreover, we have $\Phi^{2}=f\left(U^{2}\right)$, where $f(u)=m^{2} u-\ell^{2}, m>0, \ell \geqslant 0$. Hence, the second and the third formulas in (16) are simplified. The fourth and the last two formulas also become simpler:

$$
\begin{gathered}
\rho^{\prime 2}=\frac{m^{4} U^{2} U^{\prime 2}}{\left(m^{2} U^{2}-\ell^{2}\right) F_{1}\left(m^{2} U^{2}-\ell^{2}\right)}, \\
\lambda \circ \rho(s)=\int \frac{m U}{\left[m^{2} U^{2}-\ell^{2}\right]^{1 / 2}}\left[1-\frac{m^{4} U^{2} U^{\prime 2} G_{1}\left(m^{2} U^{2}-\ell^{2}\right)}{\left(m^{2} U^{2}-\ell^{2}\right) F_{1}\left(m^{2} U^{2}-\ell^{2}\right)}\right]^{1 / 2} d s, \\
\varphi(s, \tau)=\frac{\tau}{m}-\ell \int \frac{(\lambda \circ \rho)^{\prime}}{m^{2} U^{2}} d s .
\end{gathered}
$$

Now let us turn our attention to the Heisenberg space: in view of Remark 2, example (3) and Theorem 3.1, if $M^{3}$ is a Heisenberg space, then $\Phi^{2}=f\left(U^{2}\right)$, where

$$
f(u)=\left(\ell+\sqrt{1-2 \ell+m^{2} u}\right)^{2}-1, \quad m>0,0 \leqslant \ell \leqslant 1,
$$

since $\Phi^{2}=\sinh ^{2} \rho$ and $m^{2} U^{2}=\ell^{2}+\sinh ^{2} \rho-4 \ell \sinh ^{2}(\rho / 2)(0 \leqslant \ell \leqslant 1)$. Hence, we establish the following formulas for screw motion surfaces in the Heisenberg space:

$$
\begin{gathered}
\lambda \circ \rho(s) \\
=\int \frac{m U\left[\left(2(\ell-1)+2 \sqrt{1-2 \ell+m^{2} U^{2}}\right)\left(1-2 \ell+m^{2} U^{2}\right)-m^{4} U^{2} U^{\prime 2}\right]^{1 / 2}}{2\left(\sqrt{1-2 \ell+m^{2} U^{2}}+\ell-1\right) \sqrt{1-2 \ell+m^{2} U^{2}}}, \\
\varphi(s, \tau)=\frac{\tau}{m}-\int \frac{(\lambda \circ \rho)^{\prime}\left[1-\sqrt{1-2 \ell+m^{2} U^{2}}\right]}{m^{2} U^{2}} d s .
\end{gathered}
$$

We also observe that, in view again of the previous Remark 2, examples (1) (c) and (3) and (25), explicit formulas may be written either for parabolic or hyperbolic screw motion surfaces in $\mathbb{H}^{2} \times \mathbb{R}$.

\section{Complete embedded minimal and constant mean curvature surfaces in $\mathbb{H}^{2} \times \mathbb{R}$ invariant by hyperbolic screw motions}

Notice that hyperbolic screw motion surfaces in $\mathbb{H}^{2} \times \mathbb{R}$ can be studied in the same way as parabolic screw motion surfaces. Recall the definition: 
$x=e^{\varphi} \cos \rho, y=e^{\varphi} \sin \rho, t=\lambda(\rho)+\ell \varphi, 0<\rho<\pi$. Here we take the upper halfplane model for $\mathbb{H} \times\{0\}$. As in [19], we deduce that the mean curvature equation (with respect to the unit normal

$$
N=\left(1 / \sqrt{1+\sin ^{2} \rho\left(\ell^{2}+\lambda^{\prime 2}\right)}\right)\left(\sin ^{2} \rho e^{\varphi+i \rho}\left(\ell+i \lambda^{\prime}\right),-1\right)
$$

in complex notation) has the following form:

$$
\begin{aligned}
2 H\left(1+\ell^{2} \sin ^{2} \rho+\lambda^{\prime 2} \sin ^{2} \rho\right)^{3 / 2}= & -\lambda^{\prime \prime} \sin ^{2} \rho\left(1+\ell^{2} \sin ^{2} \rho\right) \\
& +\lambda^{\prime} \cos \rho \sin ^{3} \rho\left(\ell^{2}+\lambda^{\prime 2}\right)
\end{aligned}
$$

If $H$ is constant, we deduce that

$$
\frac{\lambda^{\prime}}{\sqrt{1+\ell^{2} \sin ^{2} \rho+\lambda^{\prime 2} \sin ^{2} \rho}}=2 H \cot \rho+d .
$$

Notice that, in view of (27), we deduce that the generating curve of an $H$-hyperbolic screw motion surface is given (writing $\theta=\rho$ ) by

$$
t=\lambda(\theta)=\int_{*}^{\theta} \frac{\sqrt{1+\ell^{2} \sin ^{2} \rho}(d+2 H \cot \rho)}{\sqrt{1-\sin ^{2} \rho(d+2 H \cot \rho)^{2}}} d \rho .
$$

Now observe that any hyperbolic screw motion surface obtained by (28) is stable, since it is a vertical graph given by

$$
t=\lambda(\operatorname{arccot}(x / y))+\frac{\ell}{2} \ln \left(x^{2}+y^{2}\right) .
$$

Recall that vertical translations of an $H$-vertical graph are isometric deformations of ambient space producing a foliation that ensures stability. Notice that, if $\lambda \equiv 0$ (or $d=0$ ), we obtain a complete, embedded, simply connected, stable minimal surface in $\mathbb{H}^{2} \times \mathbb{R}$, invariant by hyperbolic screw motions (with pitch $\ell$ ). Define $(R, \theta) \mapsto(R \cos \theta, R \sin \theta, \ell \ln R), R>0,0<\theta<\pi$. In fact, in view of (27) and (28), just make $\theta=\rho, R=e^{\varphi}$. This one-parameter family of minimal surfaces, as vertical graphs over the entire hyperbolic plane, is given by the following explicit nonparametric formula:

$$
t=\frac{\ell}{2} \ln \left(x^{2}+y^{2}\right), \quad y>0 .
$$

With the aid of the nonparametric Equation (4), notice that we may check that (29) yields a minimal surface. Observe now that by making $H=1 / 2$ (a downward pointing inner unit normal) and $d=0$ in (27) or (28), we obtain a complete, embedded, simply connected, stable $H$-surface in $\mathbb{H}^{2} \times \mathbb{R}$, invariant by hyperbolic screw motions (with 


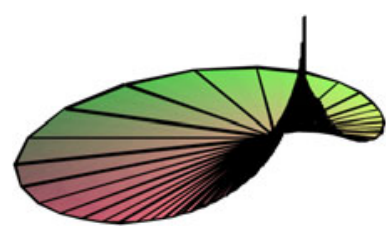

FigurE 11. The ball model for $\mathbb{H}^{2} \times\{0\}$. A complete embedded stable minimal surface $(d=0, \ell=1)$ in $\mathbb{H}^{2} \times \mathbb{R}$, invariant by hyperbolic screw motions.

pitch $\ell$ ). This surface is a vertical $H$-graph with $H=-1 / 2$ (an upward pointing inner unit normal), over the entire hyperbolic plane, given by the following explicit formula (see Figure 13):

$$
t=-\frac{\sqrt{x^{2}+y^{2}+\ell^{2} y^{2}}}{y}+\ell\left[\ln \left(\sqrt{x^{2}+y^{2}+\ell^{2} y^{2}}+\ell y\right)\right], \quad y>0 .
$$

Letting $\ell=0$ in (30) and taking the symmetric with respect to the horizontal $x y$-plane, we derive a quite simple formula for an $H$-vertical graph, over the entire hyperbolic plane $(H=1 / 2)$, invariant by hyperbolic translations:

$$
t=\frac{\sqrt{x^{2}+y^{2}}}{y}, \quad y>0 .
$$

Notice that this entire 1/2-graph (31), invariant by hyperbolic translations, has the property that the level curve $\{t=1\}$ is a geodesic and the level curves $\{t=c, c>1\}$ are equidistant curves in $\mathbb{H}^{2}$.

Of course, using (4) we can readily check that (30) and (31) are indeed $H$-vertical graphs. Very recently, Fernández and Mira [7] gave a characterization of (31) (see Figure 12).

Now, letting $H=0$ and $d=1$, and $\ell=0$ in (28), we obtain a Scherk-type minimal surface, invariant by hyperbolic translations, given by the following explicit formula:

$$
t=\ln \left(\frac{\sqrt{x^{2}+y^{2}}+y}{x}\right), \quad y>0, x>0 .
$$

Notice that this function takes infinite boundary value data on the positive $y$-axis and zero asymptotic value boundary data on the positive $x$-axis. So by applying a (horizontal) isometry of ambient space, we have the situation that we have drawn schematically in Figure 1.

We note that formula (32) was used by Collin and Rosenberg [5] in the construction of entire minimal graphs in $\mathbb{H}^{2} \times \mathbb{R}$ that are conformally the complex plane $\mathbb{C}$, disproving a conjecture by Schoen [5]. Again, in view of (4), it is easily verified that (32) yields a minimal surface. Letting $H=0$ and $\ell=0, d=1 / 2$ in (28), we obtain a complete, embedded, stable minimal surface invariant by hyperbolic translations. 


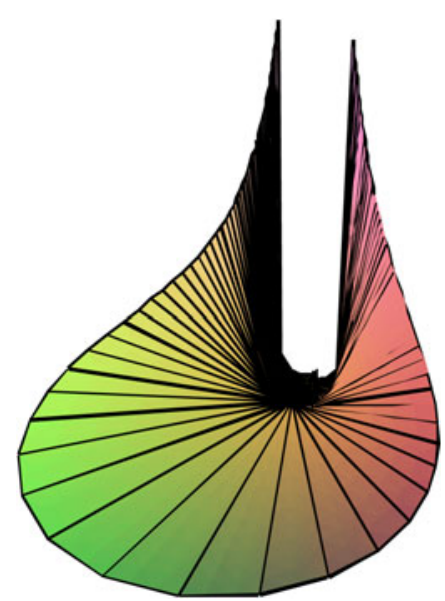

FigurE 12. The ball model for $\mathbb{H}^{2} \times\{0\}$. A complete embedded stable $H$-surface $(d=0, \ell=0)$ in $\mathbb{H}^{2} \times \mathbb{R}$, invariant by hyperbolic translations, $H=1 / 2$.

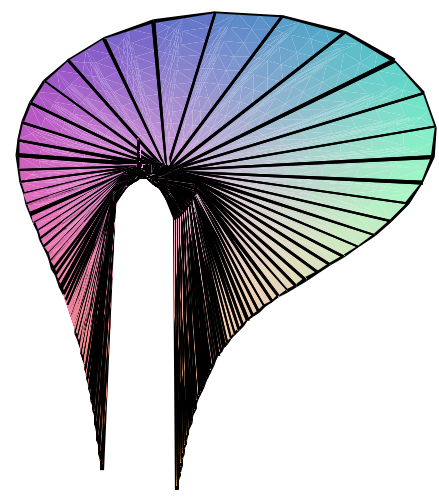

FigurE 13. The ball model for $\mathbb{H}^{2} \times\{0\}$. A complete embedded stable $H$-surface $(d=0, \ell=1)$ in $\mathbb{H}^{2} \times \mathbb{R}$, invariant by hyperbolic screw motions, $H=-1 / 2$.

Letting $H=1 / 4, \ell=0$ and $d=1 / 2$ in (28) yields a complete, embedded, stable $H$-surface invariant by hyperbolic translations. The fact that these $H$-surfaces are complete and embedded follows from the analysis of the generating curves. Other such surfaces may be constructed in the same way.

We pause now to say that the Scherk-type surface (32) (see Figure 14) can be applied to derive results for the Dirichlet problem for the minimal equation in $\mathbb{H}^{2} \times \mathbb{R}$. Very recently, Collin and Rosenberg have proved the existence of harmonic diffeomorphisms from $\mathbb{C}$ to $\mathbb{H}^{2}$ by studying complete minimal graphs in $\mathbb{H}^{2} \times \mathbb{R}$. They used the formula (32), as we noted before [5].

The following remark is due to Rosenberg. 

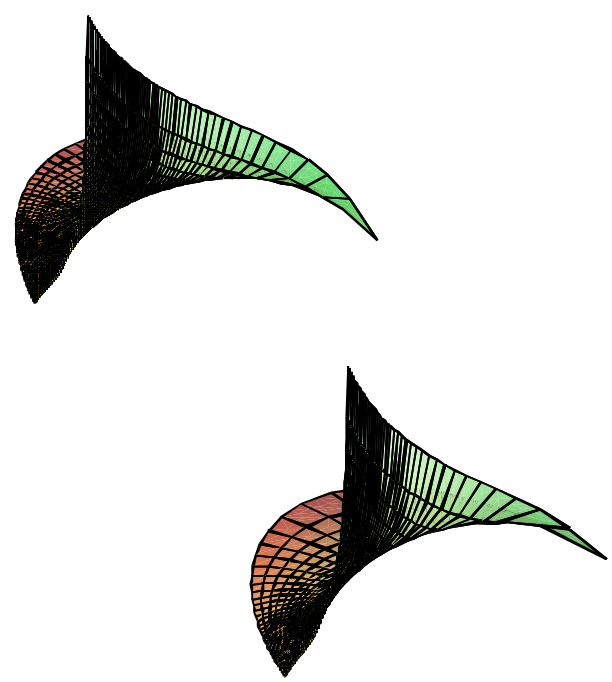

Figure 14. The ball model for $\mathbb{H}^{2} \times\{0\}$. Part of a Scherk-type minimal surface $(\ell=0, d=1)$ in $\mathbb{H}^{2} \times \mathbb{R}$, invariant by hyperbolic translations.

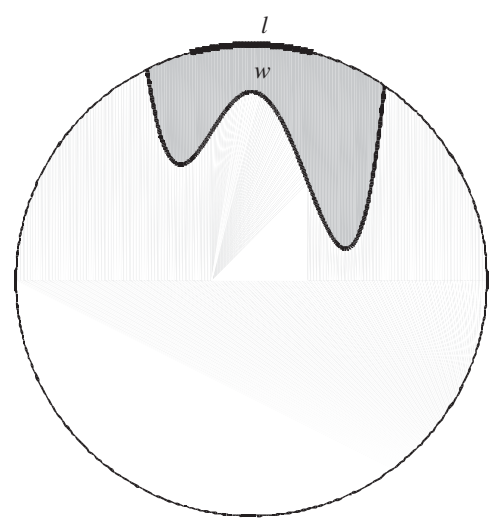

FIGURE 15. The ball model for $\mathbb{H}^{2} \times\{0\}$.

REMARK 4. Abresch and Rosenberg studied the asymptotic values of minimal graphs in $\mathbb{H}^{2} \times \mathbb{R}$ over domains in $\mathbb{H}^{2}$. In particular, they observed that there is no minimal graph $u$ in a domain $W$ of $\mathbb{H}^{2}$, taking infinite asymptotic values on an arc $l$ of the asymptotic boundary of $W$. See Figure 15 . This can be seen using the graph $S$ given by (32) (see also Figure 1) with asymptotic value $-\infty$ on a geodesic arc $g$ inside $W$ with boundary two points of $l$. Choose $g$ so that the assumed solution $u$ (which tends to infinity on $l$ ) is positive on the domain $U$ bounded by $g$ and an $\operatorname{arc}$ of $l$, see Figure 16 . Clearly, the graph of the Scherk-type surface is below $u$ where it is defined. Now translate the Scherk-type graph upwards. Then there is a first point of contact of the graph with the graph of $u$, which is impossible by the maximum principle. 


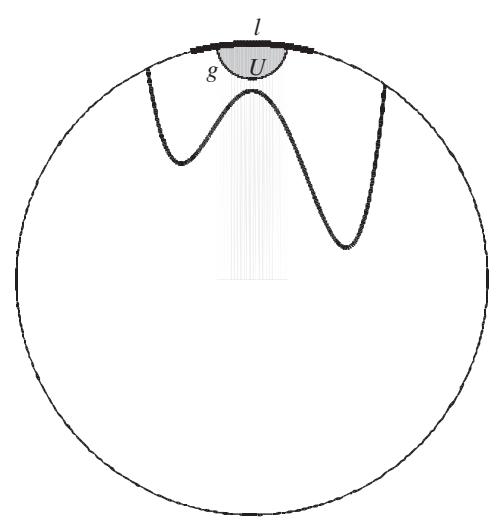

FIGURE 16. The ball model for $\mathbb{H}^{2} \times\{0\}$.

Rosenberg conjectured that there is no minimal graph over a domain in $\mathbb{H}^{2}$ with infinite asymptotic values on a set of positive measure of the circle at infinity.

We will now establish the following results for further reference.

THEOREM 4.1. Any surface invariant by parabolic screw motions in $\mathbb{H}^{2} \times \mathbb{R}$ can be parameterized locally by natural coordinates $s, \tau$. Let $S$ be such a parabolic screw motion surface. Then there exists a two-parameter family $\mathcal{F}(m, \ell), m \neq 0$, containing a surface invariant by parabolic translations, such that each element of the family is a parabolic screw motion surface isometric to $S$ given by

$$
\begin{gathered}
m^{2} U^{2}(s)=\frac{1}{y^{2}}+\ell^{2}, \\
\rho^{\prime 2}(s)=\frac{y^{2}\left(1+y^{2} \ell^{2}\right)}{1+y^{2} \ell^{2}+y^{2}\left(\lambda^{\prime}(y)\right)^{2}}, \\
\rho^{\prime 2}(s)=\frac{m^{4} U^{2} U^{\prime 2}}{\left(m^{2} U^{2}-\ell^{2}\right)^{3}}, \\
\lambda \circ \rho(s)=\int \frac{m U \sqrt{\left(m^{2} U^{2}-\ell^{2}\right)^{2}-m^{4} U^{2} U^{\prime 2}}}{\sqrt{\left(m^{2} U^{2}-\ell^{2}\right)^{3}}} d s, \\
\varphi(s, \tau)=\frac{\tau}{m}-\ell \int \frac{\sqrt{\left(m^{2} U^{2}-\ell^{2}\right)^{2}-m^{4} U^{2} U^{\prime 2}}}{m U \sqrt{\left(m^{2} U^{2}-\ell^{2}\right)^{3}}} d s .
\end{gathered}
$$

PRoOF. Using Remark 2, example (1) (b), Theorem 3.1 and Remark 3, we deduce the formulas (33) in the theorem. To see that the family contains a surface invariant by parabolic translations, we argue as follows. Looking at Equations (33), we see that if these formulas hold for some pitch $\ell \geqslant 0$, then they also hold for a pitch $\tilde{\ell}$ in the interval $[0, \ell]$, since $\left(m^{2} U^{2}-\ell^{2}\right) \geqslant 0$ and $\left(m^{2} U^{2}-\ell^{2}\right)^{2}-m^{4} U^{2} U^{\prime 2} \geqslant 0$. 
We obtain thereby a family $\mathcal{F}(m, \ell), m \neq 0$ of surfaces isometric to $S$ containing a parabolic translation surface $(\ell=0)$. This completes the proof of the theorem.

THEOREM 4.2. Any surface invariant by hyperbolic screw motions in $\mathbb{H}^{2} \times \mathbb{R}$ can be parameterized locally by natural coordinates $s, \tau$. Let $S$ be such a hyperbolic screw motion surface. Then there exists a two-parameter family $\mathcal{F}(m, \ell), m \neq 0$, containing a surface invariant by hyperbolic translations, such that each element of the family is a hyperbolic screw motion surface isometric to $S$ given by

$$
\begin{gathered}
m^{2} U^{2}(s)=\frac{1}{\sin ^{2} \rho}+\ell^{2}, \\
\rho^{\prime 2}(s)=\frac{\sin ^{2} \rho\left(1+\ell^{2} \sin ^{2} \rho\right)}{1+\ell^{2} \sin ^{2} \rho+\sin ^{2} \rho\left(\lambda^{\prime}(\rho)\right)^{2}}, \\
\rho^{\prime 2}(s)=\frac{m^{4} U^{2} U^{\prime 2}}{\left(m^{2} U^{2}-\ell^{2}\right)^{2}\left(m^{2} U^{2}-\ell^{2}-1\right)}, \\
\lambda \circ \rho(s)=\int \frac{m U \sqrt{\left(m^{2} U^{2}-\ell^{2}\right)\left(m^{2} U^{2}-\ell^{2}-1\right)-m^{4} U^{2} U^{\prime 2}}}{\left(m^{2} U^{2}-\ell^{2}\right) \sqrt{\left(m^{2} U^{2}-\ell^{2}-1\right)}} d s, \\
\varphi(s, \tau)=\frac{\tau}{m}-\ell \int \frac{\sqrt{\left(m^{2} U^{2}-\ell^{2}\right)\left(m^{2} U^{2}-\ell^{2}-1\right)-m^{4} U^{2} U^{\prime 2}}}{m U^{2}\left(m^{2} U^{2}-\ell^{2}\right) \sqrt{\left(m^{2} U^{2}-\ell^{2}-1\right)}} d s .
\end{gathered}
$$

PROOF. The proof is the same as in Theorem 4.1.

We now wish to complete the geometric description of the families of minimal and constant mean curvature parabolic screw motion surfaces given in Section 2. Given a constant $H$ satisfying $|H|<1 / 2$, we need to determine explicitly the metric of all such isometric immersions with the same mean curvature $H$. This is established by the following result.

Corollary 4.1. Let $S$ be a parabolic screw motion minimal or an H-surface invariant by parabolic screw motion with pitch $\ell$ immersed into $\mathbb{H}^{2} \times \mathbb{R}$ parameterized by natural coordinates $s, \tau$. Let $d$ be the parameter given by (8). Let us assume that $|H|<1 / 2$.

If $d=0$, then

$$
m^{2} U^{2}=e^{ \pm 2 \sqrt{1-4 H^{2}}\left(s-s_{0}\right)}+\ell^{2}
$$

If $d>0$, then

$$
\sqrt{m^{2} U^{2}-\ell^{2}}=\frac{2|H| d}{1-4 H^{2}}+\frac{d}{1-4 H^{2}} \cosh \left(\sqrt{1-4 H^{2}}\left(s-s_{0}\right)\right) .
$$

Proof. Assume that $-1 / 2<H \leqslant 0$. We combine the first three equations in Theorem 4.1 with the integral formula. After some computations we obtain

$$
\begin{aligned}
& m^{4} U^{2} U^{\prime 2}-\left(1-4 H^{2}\right)\left(m^{2} U^{2}-\ell^{2}\right)^{2}+4|H| d\left(m^{2} U^{2}-\ell^{2}\right)^{3 / 2} \\
& \quad+d\left(m^{2} U^{2}-\ell^{2}\right)=0 .
\end{aligned}
$$


Now, by making the change of variables $Z=\left(m^{2} U^{2}-\ell^{2}\right)^{1 / 2}$, we deduce the formula

$$
Z^{\prime 2}=\left(1-4 H^{2}\right) Z^{2}-4|H| d Z-d^{2} .
$$

Treating separately the cases $d=0$ and $d \neq 0$, using elementary ordinary differential equation techniques, we deduce (35) and (36), as desired. This completes the proof of the corollary.

We note that if $\ell=0$, then the Gaussian curvature $K:=-U^{\prime \prime} / U$ of a minimal parabolic screw motion immersion is $K \equiv-1$. Otherwise $K$ satisfies the inequality $-1<K<0$.

Finally, we give a description of the isometric $H$-surfaces invariant by hyperbolic screw motions.

COROLlary 4.2. Let $S$ be a hyperbolic screw motion minimal or an $H$-surface invariant by parabolic screw motion with pitch $\ell$ immersed into $\mathbb{H}^{2} \times \mathbb{R}$ parameterized by natural coordinates $s, \tau$. Let $d$ be the parameter given by (27).

(1) Assume that $|H|<1 / 2$.

(a) If $d^{2}<1-4 H^{2}$, then

$$
\begin{aligned}
& \sqrt{m^{2} U^{2}-\ell^{2}-1}= \pm \frac{\sqrt{1-4 H^{2}-d^{2}}}{1-4 H^{2}} \\
& \times\left(\sinh \sqrt{1-4 H^{2}}\left(s-s_{0}\right)\right) \pm \frac{2 H d}{1-4 H^{2}}
\end{aligned}
$$

(b) If $d^{2}>1-4 H^{2}$, then

$$
\begin{aligned}
& \sqrt{m^{2} U^{2}-\ell^{2}-1}=\frac{\sqrt{d^{2}-\left(1-4 H^{2}\right)}}{1-4 H^{2}} \\
& \times \cosh \left(\sqrt{1-4 H^{2}}\left(s-s_{0}\right)\right) \pm \frac{2 H d}{1-4 H^{2}}
\end{aligned}
$$

(c) If $d^{2}=1-4 H^{2}$, then

$$
\sqrt{m^{2} U^{2}-\ell^{2}-1}=\exp \left( \pm \sqrt{1-4 H^{2}}\left(s-s_{0}\right)\right) \pm \frac{2 H}{\sqrt{1-4 H^{2}}} .
$$

(2) Assume that $|H|=1 / 2$.

(a) If $d=0$, then

$$
\sqrt{m^{2} U^{2}-\ell^{2}-1}= \pm\left(s-s_{0}\right) .
$$

(b) If $|H|>1 / 2$, then

$$
\sqrt{m^{2} U^{2}-\ell^{2}-1}= \pm\left[\frac{d}{2}\left(s-s_{0}\right)^{2}-\frac{1-d^{2}}{d^{2}}\right] .
$$


(3) Assume that $|H|>1 / 2$. Then

$$
\begin{aligned}
\sqrt{m^{2} U^{2}-\ell^{2}-1}= & \pm \frac{\sqrt{d^{2}+4 H^{2}-1}}{4 H^{2}-1} \sin \left(\sqrt{4 H^{2}-1}\left(s-s_{0}\right)\right) \\
& \pm \frac{2 H d}{4 H^{2}-1} .
\end{aligned}
$$

PROOF. As in Corollary 4.1, by applying the relations derived in Theorem 4.2 together with the first integral formula (27), we infer the differential equation

$$
Z^{\prime 2}=Z^{2}\left(1-4 H^{2}\right) \pm 4 H d Z+1-d^{2}
$$

where $Z=\sqrt{m^{2} U^{2}-\ell^{2}-1}$. Now, by working with elementary ordinary differential equations, we deduce the formulas in the corollary, as desired.

\section{Associate and conjugate parabolic and hyperbolic screw motion immersions}

Let $M^{2}$ be a two-dimensional Riemannian manifold. Let $(x, y, t)$ be local coordinates in $M^{2} \times \mathbb{R}$, where $z=x+i y$ are conformal coordinates on $M^{2}$ and $t \in \mathbb{R}$. Let $\sigma^{2}|d z|^{2}$, be the conformal metric in $M^{2}$; hence $d s^{2}=\sigma^{2}|d z|^{2}+d t^{2}$ is the metric in the product space $M^{2} \times \mathbb{R}$. Let $\Omega \subset \mathbb{C}$ be a planar domain, $w=$ $u+i v \in \Omega$. We recall that if $X: \Omega \rightarrow M^{2} \times \mathbb{R}, w \mapsto(h(w), f(w)), w \in \Omega$ is a conformal minimal immersion with induced metric $d s^{2}=\mu^{2}|d w|^{2}$, then $h: \Omega \subset \mathbb{C} \rightarrow$ $\left(M^{2}, \sigma^{2}|d z|^{2}\right), w \mapsto h(w)$ is a harmonic map, that is, it satisfies

$$
h_{w \bar{w}}+2 \frac{\sigma_{z}}{\sigma} h_{w} h_{\bar{w}}=0
$$

see, for instance, [19]. We recall also that for any harmonic map $h: \Omega \subset \mathbb{C} \mapsto M^{2}$ there exists a related Hopf holomorphic function given by

$$
\phi=(\sigma \circ h)^{2} h_{w} \bar{h}_{w}
$$

see $[20,22]$. Toubiana and the current author have introduced the notions of associate and conjugate immersions in [19], following a work in progress with Hauswirth [11]. Namely, two conformal isometric immersions $X, \tilde{X}: \Omega \hookrightarrow \mathbb{H}^{2} \times \mathbb{R}$ are said to be associate if the Hopf functions satisfy the relation $\widetilde{\phi}=e^{i \theta} \phi$. If $\widetilde{\phi}=-\phi$, then the two immersions are said to be conjugate. Daniel has given an alternative and equivalent definition [2].

As we said in the introduction, Toubiana and the current author proved that any two minimal isometric screw motion immersions in $\mathbb{H}^{2} \times \mathbb{R}$ and $\mathbb{S}^{2} \times \mathbb{R}$ are associate. The same authors proved that, in $\mathbb{H}^{2} \times \mathbb{R}$, a catenoid is conjugate to a helicoid of pitch $\ell<1$.

We will now prove that two minimal isometric parabolic screw motion immersions are associate. 
THEOREM 5.1. Any two minimal isometric parabolic screw motion immersions into $\mathbb{H}^{2} \times \mathbb{R}$ are associate. Furthermore, a helicoid of pitch $\ell=1$ is conjugate to a surface invariant by parabolic translations, that is, it is conjugate to a Daniel-Hauswirth minimal surface.

We note that Daniel [2] proved the second part of the above theorem by another approach.

PROOF. Let us now take natural coordinates $s, \tau$ so that the induced metric is given by $d \mu^{2}=d s^{2}+U^{2} d \tau^{2}$, see Theorem 4.1. Therefore, we have natural conformal coordinates $v+i \tau$, where $v=\int 1 / U d s$. Of course, the induced metric becomes $d \mu^{2}=U^{2}\left(d v^{2}+d \tau^{2}\right)$. We may therefore compute the Hopf function $\phi$ for parabolic screw motion minimal immersions. After a somewhat long computation, working as in [19], we obtain the relations

$$
\begin{aligned}
4 \operatorname{Re} \phi & =\frac{1}{m^{2}}\left[\ell^{2}-\frac{\lambda^{\prime 2}}{1+r^{2}\left(\ell^{2}+\lambda^{\prime 2}\right)}\right], \\
\operatorname{Im} \phi & =0 \quad \text { if } \ell=0, \\
4 \operatorname{Re} \phi & =\frac{\ell^{2}}{m^{2}}-\frac{4 m^{2}}{\ell^{2}}(\operatorname{Im} \phi)^{2} \quad \text { if } \ell \neq 0 .
\end{aligned}
$$

Using (43) and (8), we deduce that $\phi$ is given by

$$
m^{2} \phi=\frac{\ell^{2}}{4}-\frac{d^{2}}{4}+i \frac{\ell d}{2} \quad(d \geqslant 0)
$$

Thus

$$
16|\phi|^{2}=\left(\frac{\ell^{2}}{m^{2}}+\frac{d^{2}}{m^{2}}\right)^{2} .
$$

Now two isometric minimal parabolic screw motion immersions have the metric given by Corollary 4.1, making $H=0$; that is either

$$
m^{2} U^{2}=e^{2\left(s-s_{0}\right)}+\ell^{2} \quad \text { if } d=0,
$$

or

$$
m^{2} U^{2}=\frac{d^{2}}{2} \cosh \left(2\left(s-s_{0}\right)\right)+\ell^{2}+\frac{d^{2}}{2} \quad \text { if } d \neq 0 .
$$

In view of (44) and (45), the absolute value of their Hopf functions are the same, and hence they are associate as well. Now, on account of [19] the helicoid $(d=0)$ of pitch $\ell=1$ has metric given by $U^{2}=\left(1 / m^{2}\right) \cosh ^{2}\left(s-s_{0}\right)$ and Hopf function given by $\phi=\left(1 / 4 m^{2}\right)$. On the other hand, in view of Corollary 4.1, the Daniel-Hauswirth minimal surface $(\ell=0, m=1)$ has metric given by $U^{2}=d^{2} \cosh ^{2}\left(s-s_{0}\right)$ and Hopf function given by $\phi=-d^{2} / 4$. Thus we have that if $d^{2} m^{2}=1$, then they are conjugate, as desired. This completes the proof of the theorem. 
Next, we will prove that the nonisometric deformation of the hyperbolic plane given by tilted Euclidean plane (9), invariant by parabolic screw motions with pitch $\ell=1$, is conjugate to the Scherk-type minimal surface, invariant by hyperbolic translations given by (32). See Figures 5 and 14.

Let $d$ be the parameter given in (27).

THEOREM 5.2. The conjugate of the Scherk-type minimal surface invariant by hyperbolic translations is the minimal surface generated by a horizontal line invariant by parabolic screw motions with pitch $\ell=1$. If $d^{2}<1$, any two minimal isometric hyperbolic screw immersions in this family are associate. The same holds if either $d^{2}=1$ or $d^{2}>1$. To each minimal hyperbolic screw motion immersion in the family $d^{2}<1$, there exists a minimal isometric nonassociate hyperbolic screw motion immersion in the family $d^{2}>1$. Furthermore, each parabolic screw motion surface is associate to a hyperbolic screw motion surface. Any helicoid with pitch $\ell>1$ is conjugate to a minimal surface invariant by hyperbolic translations $(\ell=0$ and $\left.d^{2}>1\right)$.

We remark that the last part of theorem (about the helicoid) is a result of Daniel [2]. We will give an alternative proof.

PROOF. We first observe that the structure of the proof is the same as in Theorem 5.1. On account of Theorem 4.2, we can deduce the following relations:

$$
\begin{gathered}
4 \operatorname{Re} \phi=\frac{1}{m^{2}}\left[\ell^{2}-\frac{\lambda^{\prime 2}}{1+\sin ^{2} \rho\left(\ell^{2}+\lambda^{\prime 2}\right)}\right], \\
\operatorname{Im} \phi=0 \quad \text { if } \ell=0, \\
4 \operatorname{Re} \phi=\frac{\ell^{2}}{m^{2}}-\frac{4 m^{2}}{\ell^{2}}(\operatorname{Im} \phi)^{2} \quad \text { if } \ell \neq 0 .
\end{gathered}
$$

Using (46) and (27), we deduce that $\phi$ is given by

$$
m^{2} \phi=\frac{\ell^{2}}{4}-\frac{d^{2}}{4}+i \frac{\ell d}{2} \quad(d \geqslant 0) .
$$

Hence

$$
16|\phi|^{2}=\left(\frac{\ell^{2}}{m^{2}}+\frac{d^{2}}{m^{2}}\right)^{2} .
$$

It now follows from (40) and (47) that the metric and the Hopf function of the Scherk-type minimal surface (32) are given by $m^{2} U^{2}=e^{2\left(s-s_{0}\right)}+1, \phi=-\left(1 / 4 m^{2}\right)$, respectively. On the other hand, from (35) and (44) the metric and the Hopf function of the nonisometric minimal deformation of the hyperbolic plane (9) with pitch $\ell=1$ are given by $m^{2} U^{2}=e^{2\left(s-s_{0}\right)}+1, \phi=\left(1 / 4 m^{2}\right)$, respectively. Hence the immersions are conjugate. This proves the first part of the theorem. 
Let us first assume that $d^{2}<1$. According to (38), the metrics of two isometric minimal immersions are given by

$$
\begin{aligned}
m^{2} U^{2} & =\left(1-d^{2}\right) \sinh ^{2}\left(s-s_{0}\right)+\ell^{2}+1 \\
& =\left(1-d^{2}\right) \cosh ^{2}\left(s-s_{0}\right)+d^{2}+\ell^{2} .
\end{aligned}
$$

Thus, on account of (47), any two such isometric immersions are associate. Second, if $d^{2}=1$, we infer from (40) and (47) the same result. Analogously, if $d^{2}>1$, the metric is given by

$$
\begin{aligned}
m^{2} U^{2} & =\frac{d^{2}-1}{2} \cosh \left(2\left(s-s_{0}\right)\right)+\ell^{2}+\frac{d^{2}+1}{2} \\
& =\left(d^{2}-1\right) \cosh ^{2}\left(s-s_{0}\right)+\ell^{2}+1 .
\end{aligned}
$$

Now take a minimal hyperbolic screw motion immersion in the first family $\left(d^{2}<1\right)$, setting $m=m_{\hbar_{1}}, d=d_{\hbar_{1}}, \ell=\ell_{\hbar_{1}}$ in (48). We see that there exists a minimal hyperbolic screw motion immersion $(m, d, \ell)$ in the third family $\left(d^{2}>1\right)$, see (49), by setting

$$
\frac{d^{2}-1}{m^{2}}=\frac{1-d_{\hbar_{1}}^{2}}{m_{\hbar_{1}}^{2}}, \quad \frac{\ell^{2}+1}{m^{2}}=\frac{d_{\hbar_{1}}^{2}+\ell_{\hbar_{1}}^{2}}{m_{\hbar_{1}}^{2}} .
$$

Let $\phi_{\hbar_{1}}$ and $\phi$ be their Hopf functions, respectively. Notice that, using (47) and (50), we deduce the strict inequality $\left|\phi_{\hbar_{1}}\right|<|\phi|$, and hence these isometric immersions are nonassociate.

Notice that any minimal parabolic screw motion immersion $S=S\left(d_{\mathcal{P}}, m_{\mathcal{P}}, \ell_{\mathcal{P}}\right)$, with $d_{\mathcal{P}}^{2} \neq 0$, has metric given by (36), setting $H=0$. In view of (49), (44) and (47), by setting

$$
\frac{d^{2}-1}{m^{2}}=\frac{d_{\mathcal{P}}^{2}}{m_{\mathcal{P}}^{2}}, \quad \frac{\ell^{2}+1}{m^{2}}=\frac{\ell_{\mathcal{P}}^{2}}{m_{\mathcal{P}}^{2}},
$$

we obtain a minimal hyperbolic screw motion surface in the third family associate to $S$. In the same way, we deduce that any minimal parabolic screw motion immersion $\left(d_{\mathcal{P}}, m_{\mathcal{P}}, \ell_{\mathcal{P}}\right)$, with $d_{\mathcal{P}}^{2}=0$, is associate to a minimal hyperbolic screw motion surface in the second family $\left(d^{2}=1\right)$. Therefore, we conclude that each parabolic screw motion surface is associate to a hyperbolic screw motion surface.

Finally, consider a minimal surface invariant by hyperbolic translations with parameters $\left(d^{2}>1, m_{\hbar}, \ell=0\right)$, metric given by (39) or (49) (with $m=m_{\hbar}, \ell=0$, $d^{2}>1$ ) and Hopf function given by $\phi_{\hbar}=-d^{2} / 4 m_{\hbar}^{2}$. Owing to [19], the helicoid with pitch $\ell_{\mathcal{H}}$ has metric given by

$$
m_{\mathcal{H}}^{2} U^{2}=\frac{1}{2} \cosh \left(2\left(s-s_{0}\right)\right)+\ell_{\mathcal{H}}^{2}-\frac{1}{2}
$$


and Hopf function given by $\phi=\ell_{\mathcal{H}}^{2} / 4 m_{\mathcal{H}}^{2}$. Therefore, if $\left(d^{2}-1\right) / 2 m_{\hbar}^{2}=1 / 2 m_{\mathcal{H}}^{2}$, and $\left(d^{2}+1\right) / 2 m_{\hbar}^{2}=\left(2 \ell_{\mathcal{H}}^{2}-1\right) / 2 m_{\mathcal{H}}^{2}$, we deduce that any helicoid of pitch $\ell_{\mathcal{H}}>1$ is conjugate to a minimal surface invariant by hyperbolic translations, as desired. This completes the proof of the theorem.

\section{References}

[1] U. Abresch and H. Rosenberg, 'A Hopf differential for constant mean curvature surfaces in $S^{2} \times R$ and $H^{2} \times R$ ', Acta Math. 193(2) (2004), 141-174.

[2] B. Daniel, 'Isometric immersions into $\mathbb{S}^{n} \times \mathbb{R}$ and $\mathbb{H}^{n} \times \mathbb{R}$ and applications to minimal surfaces', in: Prépublications, Vol. 375 (Institut de Math. de Jussieu, 2004).

[3] E. Bour, 'Mémoire sur la déformation des surfaces', J. Ecole Pol. 39 (1862), 1-148.

[4] M. P. Do Carmo and M. Dajczer, 'Helicoidal surfaces with constant mean curvature', Tohoku Math. J. 34 (1982), 425-435.

[5] P. Collin and H. Rosenberg, 'Construction of harmonic diffeomorfisms and minimal graphs', Preprint (arXiv: math.DG/0701547v1).

[6] U. Dierkes, S. Hildebrandt, A. Küster and O. Wohlrab, Minimal Surfaces, Vol. I: Boundary Value Problems and Vol. II Boundary Regularity (Grundlehren Der Mathematischen Wissenschaften) 295 and 296 (Springer, Berlin, 1992).

[7] I. Fernández and P. Mira, 'Harmonic maps and constant mean curvature surfaces in $\mathbb{H}^{2} \times \mathbb{R}^{\text {,', }}$ Amer. J. Math. 129(4) (2007), 1145-1181.

[8] - 'A characterization of constant mean curvature surfaces in homogeneous 3-manifold,', Differential Geom. Appl. 25(3) (2007), 281-289.

[9] — 'Holomorphic quadratic differentials and the Bernstein problem in Heisenberg space', Preprint.

[10] L. Hauswirth, 'Generalized Riemann examples in three-dimensional manifolds', Pacific J. Math. 224(1) (2006), 91-117.

[11] L. Hauswirth, R. Sa Earp and E. Toubiana, 'Associate and conjugate minimal immersions in $M^{2} \times R$ ', Tohoku Math. J. 60 (2008), 267-286.

[12] L. Hauswirth, H. Rosenberg and J. Spruck, 'Infinite boundary value problems for constant mean curvature graphs in $\mathbb{H}^{2} \times \mathbb{R}$ and $\mathbb{S}^{2} \times \mathbb{R}$ ', http://www.math.jhu.edu/ js/jsfinal.pdf.

[13] W. Meeks III and H. Rosenberg, 'The theory of minimal surfaces in $M \times \mathbb{R}$ ', Comment. Math. Helv. 80(4) (2005), 811-858.

[14] B. Nelli and H. Rosenberg, 'Minimal surfaces in $\mathbb{H}^{2} \times \mathbb{R}^{\prime}$, Bull. Braz. Math. Soc. 33 (2002), 263-292.

[15] - 'Global properties of constant mean curvature surfaces in $\mathbb{H}^{2} \times \mathbb{R}^{\prime}$, Pacific J. Math. 226(1) (2006), 137-152.

[16] J. Ordóñes, 'Superfícies helicoidais com curvatura constante no espaço de formas tridimensionais', Doctoral Thesis, PUC-Rio, 1995.

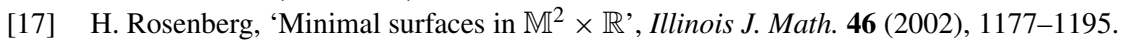

[18] R. Sa Earp and E. Toubiana, 'Existence and uniqueness of minimal graphs in hyperbolic space', Asian J. Math. 4 (2000), 669-694.

[19] ' 1323-1362.

[20] R. Schoen and S. T. Yau, 'Lectures on harmonic maps', Conference Proceedings and Lecture Notes in Geometry and Topology, II (International Press, Cambridge, MA, 1997).

[21] J. Spruck, 'Interior gradient estimates and existence theorems for constant mean curvature graphs

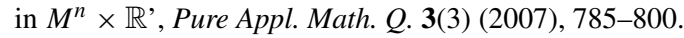

[22] T. Y. H. Wan, 'Constant mean curvature surface, harmonic maps, and universal Teichmüller space', J. Differential Geom. 35 (1992), 643-657. 
RICARDO SA EARP, Departamento de Matemática,

Pontifícia Universidade Católica do Rio de Janeiro, Rio de Janeiro, 22453-900 RJ, Brazil

e-mail: earp@mat.puc-rio.br 